\title{
Resolution Sensitivity of Cyclone Climatology over Eastern Australia Using Six Reanalysis Products*
}

\author{
Alejandro Di LuCA AND JASOn P. Evans \\ Climate Change Research Centre, Faculty of Science, University of New South Wales, Sydney, Australia \\ ACACia PePler, Lisa Alexander, And Daniel Argüeso \\ Climate Change Research Centre, ARC Centre of Excellence for Climate System Science, University of New South \\ Wales, Sydney, Australia
}

(Manuscript received 17 September 2014, in final form 5 June 2015)

\begin{abstract}
The climate of the eastern seaboard of Australia is strongly influenced by the passage of low pressure systems over the adjacent Tasman Sea due to their associated precipitation and their potential to develop into extreme weather events. The aim of this study is to quantify differences in the climatology of east coast lows derived from the use of six global reanalyses. The methodology is explicitly designed to identify differences between reanalyses arising from differences in their horizontal resolution and their structure (type of forecast model, assimilation scheme, and the kind and number of observations assimilated). As a basis for comparison, reanalysis climatologies are compared with an observation-based climatology. Results show that reanalyses, specially high-resolution products, lead to very similar climatologies of the frequency, intensity, duration, and size of east coast lows when using spatially smoothed (about $300-\mathrm{km}$ horizontal grid meshes) mean sea level pressure fields as input data. Moreover, at these coarse horizontal scales, monthly, interannual, and spatial variabilities appear to be very similar across the various reanalyses with a generally stronger agreement between winter events compared with summer ones. Results also show that, when looking at cyclones using reanalysis data at their native resolution (approaching $50-\mathrm{km}$ grid spacing for the most recent products), uncertainties related to the frequency, intensity, and size of lows are very large and it is not clear which reanalysis, if any, gives a better description of cyclones. Further work is needed in order to evaluate the usefulness of the finescale information in modern reanalyses and to better understand the sources of their differences.
\end{abstract}

\section{Introduction}

Cyclones are important meteorological phenomena that strongly influence both the day-to-day weather and the climate of many regions around the world. At the surface, cyclones are characterized by a region of low pressure, are frequently associated with cloudiness and precipitation, and have the potential to generate

\footnotetext{
* Supplemental information related to this paper is available at the Journals Online website: http://dx.doi.org/10.1175/JCLI-D-1400645.s1.

Corresponding author address: Alejandro Di Luca, Gate 11 Botany St, Library Walk Level 4, Mathews Building, University of New South Wales, Sydney NSW 2052, Australia.

E-mail: a.diluca@unsw.edu.au
}

extreme weather events (e.g., strong winds, large ocean waves, and/or heavy rain). The eastern seaboard of Australia is influenced by the passage of various types of low pressure systems, which can have distinctly different dynamics. These include large extratropical cyclones embedded in or cut off from the midlatitude westerlies, as well as decaying or transitioning tropical cyclones. Additionally, cyclones can develop within preexisting surface troughs directly off the coast, which can intensify very rapidly and form a particular forecasting challenge. Cyclones that either cross or form over the Tasman Sea adjacent to the Australian coast have been designated in a number of articles as "east coast lows" (ECLs) or "east coast cyclones" (Speer et al. 2009; Browning and Goodwin 2013; Pepler and Coutts-Smith 2013).

As extensively discussed by Mills et al. (2010), a key component in the development/intensification of some 
TABLE 1. Period and approximate area of analysis together with the number of ECL events per year and unit area for some of the previous ECLs datasets.

\begin{tabular}{llccr}
\hline & \multicolumn{1}{c}{ Period } & \multicolumn{1}{c}{ Area (lat-lon) } & Area $\left(10^{6} \mathrm{~km}^{2}\right)$ & $\mathrm{No.} \mathrm{of} \mathrm{events}\left(\mathrm{yr}^{-1} \mathrm{~km}^{-2}\right)$ \\
\hline Holland et al. (1987) & $1970-85$ & $20^{\circ}-40^{\circ}, \sim 144^{\circ}-149^{\circ}$ & 1.07 & 1.6 \\
Hopkins and Holland (1997) & $1958-92$ & $20^{\circ}-40^{\circ}, \sim 144^{\circ}-149^{\circ}$ & 1.07 & 2.2 \\
Speer et al. (2009) & $1970-2006$ & $25^{\circ}-40^{\circ}, 145^{\circ}-160^{\circ}$ & 2.34 & 9.8 \\
Browning and Goodwin (2013) & $1979-2011$ & $20^{\circ}-40^{\circ}, 145^{\circ}-162^{\circ}$ & 3.63 & 4.5 \\
\hline
\end{tabular}

ECLs is the vorticity advection associated with an intense trough or a cutoff low at upper levels over southeastern Australia. Although this large-scale baroclinic forcing can certainly be well reproduced by numerical weather prediction (NWP) models and even by coarserresolution global climate models (GCMs) (Mills et al. 2010; Dowdy et al. 2013), intensification mechanisms related with the thermal structure that develops around the low center require relatively high horizontal resolutions, which may lead to such systems being poorly represented in GCMs.

Based on various definitions of what an ECL represents, a number of ECL climatologies have been presented in the literature (Table 1). Differences in the number of ECLs across the several datasets can arise from a variety of sources such as the quality and temporal-spatial resolution of the input data, the method used to identify ECLs, and the particular way that ECLs are defined. While some authors have incorporated all lows satisfying a broad definition such as "any system with closed cyclonic circulation at sea level, which forms in a maritime environment between $20^{\circ}$ and $40^{\circ} \mathrm{S}$ and within $500 \mathrm{~km}$ of the eastern coastline of Australia" (Hopkins and Holland 1997), others have identified a particular class (i.e., a subgroup) of cyclones that share either some physical characteristics such as the direction of movement or a minimum intensity/ duration or that have the potential to produce significant damage (e.g., Holland et al. 1987; Hopkins and Holland 1997; Browning and Goodwin 2013).

Speer et al. (2009, hereinafter SP2009) developed a database of ECLs based on a subjective analysis of various datasets available at the Australian Bureau of Meteorology including 0000 UTC charts of mean sea level pressure (MSLP), station data, and satellite imagery. They found a total of 830 events with 589 of them having some associated precipitation over the eastern Australian coast. Although entirely based on observations, the SP2009 dataset presents two drawbacks. First, the use of a single chart per day (MSLP at 0000 UTC) to identify ECLs may lead to an underestimation of the number of lows because, as already discussed by Hopkins and Holland (1997), ECLs can sometimes be relatively small in size and duration. Second, the subjective character of the identification process makes it difficult to extend the database and constitutes an extra source of uncertainty when comparing statistics from different periods.

More recently, automated cyclone detection and tracking methods have been applied to study ECLs using gridded MSLP fields derived from reanalyses (e.g., Browning and Goodwin 2013; Pepler and Coutts-Smith 2013). Notably, Dowdy et al. (2013) quantified the risk of ECL formation using a large-scale diagnostic based on the geostrophic vorticity calculated at $500-\mathrm{hPa}$ pressure surface. They compared results from three reanalyses and three GCMs, and found large similarities between the climatologies regarding their seasonal, annual, and spatial variability. They also showed that the geostrophic vorticity thresholds used to identify favorable condition for ECL development are strongly dependent on the horizontal resolution of the vorticity fields.

Partly motivated through the Eastern Seaboard Climate Change Initiative (ESCCI; http://climatechange. environment.nsw.gov.au/About-climate-change-in-NSW/ Evidence-of-climate-change/Eastern-seaboard-climatechange-initiative) on ECLs, a number of projects are currently underway to better understand the development and impacts of ECLs and how their frequency, duration, and intensity may change in the future. To tackle any of these objectives and particularly to quantify likely future changes in ECLs statistics, a climatology of ECLs over the recent past climate based on an automatic algorithm that can be applied to any gridded dataset (i.e., based on observations and/or simulations) is needed.

Reanalyses of observations constitute a very useful source of data to study ECLs because of their homogenous nature in both space and time and also because they are arguably our best estimate of the 3D atmospheric state. Reanalyses have been extensively used to provide information on the mean properties and variability of extratropical cyclones (e.g., Hodges et al. 2011; Simmonds and Keay 2000; Tilinina et al. 2013, and references therein). Moreover, in recent years, a new generation of high-resolution (horizontal spacing approaching $50 \mathrm{~km}$ ) reanalysis products has been made available, providing the opportunity to extend the study 
of cyclones into subsynoptic scales. For instance, Hodges et al. (2011) compared the climatology of extratropical cyclones in both hemispheres based on four recent reanalyses, namely ERA-Interim, NASA MERRA, NCEP CFSR, and JRA-25. Overall, they found that the new reanalyses agreed better between each other compared to the old lower-resolution reanalyses, particularly over the Southern Hemisphere (SH) where they now show comparable agreement to Northern Hemisphere $(\mathrm{NH})$ results. They argued that these improvements are mostly coming from developments in the new data assimilation schemes that allow the extraction of more information from the available observations. Tilinina et al. (2013) also studied the climatology of extratropical cyclones in the $\mathrm{NH}$ based on five different reanalyses using a single cyclone tracking algorithm. They found that the total number of cyclones depends strongly on the horizontal resolution of the data and consequently found some significant differences between the climatologies obtained using the old and new reanalyses. They also found that the NASA MERRA reanalysis produces deeper and more rapidly intensifying cyclones than any other reanalysis and that the interannual variability of the number of cyclones is well correlated across the various reanalyses.

The aim of this study is to use six reanalyses, including the new higher-resolution generation, to quantify the dependence of various characteristics of maritime ECLs (e.g., frequency, intensity, size, and duration) on two factors:

- the "quality" of the dataset, assessed by comparing ECLs derived from the different reanalyses after they were regridded to similar horizontal resolutions, and

- the horizontal resolution of MSLP fields, assessed by comparing the resulting ECL climatology for a given reanalysis after being regridded at various resolutions.

The use of various reanalyses allows quantification of the uncertainty of the estimated ECL characteristics, thus making an important contribution to the previous ECL datasets that were generally based on the use of a single source. Furthermore, the relatively high spatial resolution of the new generation of reanalyses provides the opportunity to include mesoscale cyclones in the analysis. This provides a reference dataset to evaluate high-resolution climate simulations. In a future study, a 12-member Regional Climate Model ensemble generated as part of the New South Wales/Australian Capital Territory Regional Climate Modeling (NARCliM) project will be evaluated along with their future projections (Evans et al. 2014).

In this article, we make use of a single identification and tracking algorithm and so our results do not include those uncertainties coming from the choice of the algorithm. The impact of the identification and tracking algorithm, including the one used in this analysis, was investigated by Pepler et al. (2015). This study compared four different tracking schemes using the same input data and found that, although different methods may show some discrepancies on the seasonality of events, they showed a consistent picture across the various schemes when considering deep, long-lived cyclones over the ocean compared with shorter-lived, shallower cyclones.

The paper is structured as follows: in section 2 the datasets are briefly described, highlighting those differences that may lead to changes in ECL statistics; in section 3, the methodology is discussed including a description of the identification and tracking algorithm. Section 4 shows results of the mean characteristics, distributions, and variability of ECLs according to their frequency, intensity, and mean durations. Finally a summary and conclusions of the work are presented in section 5 .

\section{Datasets}

Six different although not independent sources of MSLP data are used in this study to identify ECLs. All datasets consist of the reanalysis of observations using a frozen global data assimilation system and for all of them MSLP fields are derived from the standard surface pressure fields. A brief description of each reanalysis product is given below and their main characteristics are presented in Table 2. We also include below a short description of the SP2009 ECL climatology use in this study as a basis for comparison.

\section{a. NCEP1}

The NCEP-NCAR reanalysis 1 project (hereafter NCEP1; Kalnay et al. 1996) was produced by cooperation between the National Centers for Environmental Prediction and the National Center for Atmospheric Research. It uses the T62 NCEP global spectral model (equivalent to a horizontal grid spacing of about $210 \mathrm{~km}$ ) with 28 sigma vertical levels. The NCEP1 system uses a three-dimensional variational (3Dvar) data assimilation scheme consisting of a spectral statistical interpolation system. MSLP fields are available from 1948 to the present every $6 \mathrm{~h}$ and the analyses are performed four times per day at 0000, 0600, 1200 and 1800 UTC.

\section{b. NCEP2}

The NCEP-DOE reanalysis 2 (NCEP2; Kanamitsu et al. 2002) was generated by the U.S. National Centers for Environmental Prediction and the Department of 
TABLE 2. Temporal $(\Delta t)$ and horizontal $(\Delta x)$ spacing of the available data together with the type of model and the kind of assimilation scheme for the six reanalyses used in the study. The fourth column shows those times when the analyses are produced.

\begin{tabular}{|c|c|c|c|c|c|c|}
\hline Dataset & $\Delta t(\mathrm{~h})$ & $\Delta y$ by $\Delta x(\mathrm{~km})$ & Analysis (times) & Atmospheric model type & $\begin{array}{c}\text { Ocean model } \\
\text { type }\end{array}$ & $\begin{array}{c}\text { Assimilation } \\
\text { scheme }\end{array}$ \\
\hline MERRA & 1 & $\sim 55 \times 60$ & $0000,0600,1200,1800$ UTC & Finite volume $\left(2 / 3^{\circ} \times 1 / 2^{\circ}\right)$ & No & 3Dvar \\
\hline CFSR & 1 & $\sim 45 \times 55$ & 0000, 0600, 1200, $1800 \mathrm{UTC}$ & Spectral (T382) & $\begin{array}{l}\text { Finite difference } \\
\left(1 / 2^{\circ} \times 1 / 2^{\circ}\right)\end{array}$ & 3Dvar \\
\hline ERA-I & 6 & $\sim 80 \times 70$ & 0000 and $1200 \mathrm{UTC}$ & Spectral (T255) & No & 4Dvar \\
\hline JRA-55 & 6 & $\sim 55 \times 55$ & 0000, 0600, 1200, 1800 UTC & Spectral (T319) & No & 4Dvar \\
\hline NCEP1 & 6 & $\sim 270 \times 230$ & 0000, 0600, 1200, 1800 UTC & Spectral (T62) & No & 3Dvar \\
\hline NCEP2 & 6 & $\sim 270 \times 230$ & $0000,0600,1200,1800$ UTC & Spectral (T62) & No & 3Dvar \\
\hline
\end{tabular}

Energy and constitutes an improved version of the NCEP1 reanalysis. It uses an updated version of the atmospheric model but the same spatial (T62), vertical (28 sigma levels), and temporal (6h) resolution as the NCEP1 reanalysis. The system also uses similar raw observational data although several improvements and corrections were applied particularly to the preprocessing of the data. NCEP2 reanalysis covers the period from 1 January 1979 to the present and the analyses are performed at the same times as NCEP1.

\section{c. ERA-Interim}

ERA-Interim (hereinafter ERA-I; Dee et al. 2011) constitutes a global atmospheric reanalysis provided by the European Centre for Medium-Range Weather Forecasts (ECMWF). ERA-I uses a T255 horizontal resolution, which corresponds to approximately $79-\mathrm{km}$ spacing on a reduced Gaussian grid and a total of 60 sigma-pressure levels. ERA-I also uses a more sophisticated data assimilation scheme (4Dvar) and assimilates a greater number of observations leading to a general improvement in the representation of the hydrological cycle and a better temporal consistency on a range of time scales (Dee et al. 2011) compared with its predecessor the ERA-40 reanalysis. ERA-I MSLP fields are available globally every $6 \mathrm{~h}$ from 1 January 1979 onward. The data assimilation scheme is applied every $12 \mathrm{~h}$, at 0000 and $1200 \mathrm{UTC}$, and so MSLP fields valid at 0600 and 1800 correspond to model forecasts.

\section{d. $C F S R$}

The NCEP Coupled Forecast System (CFS) Reanalysis (CFSR; Saha et al. 2010) uses the NCEP CFS and constitutes the only reanalysis using an atmosphereocean coupled model. The atmospheric component is a spectral model at a resolution of T382 (about $38 \mathrm{~km}$ ) with 64 hybrid vertical levels and the Geophysical Fluid Dynamics Laboratory (GFDL) Modular Ocean Model, version 4p0d (Griffies et al. 2004), which is a finitedifference model with horizontal grid spacings of $1 / 2^{\circ}$ in longitude and between $1 / 4^{\circ}$ and $1 / 2^{\circ}$ in latitude with
40 levels in the vertical. For the atmosphere, the NCEP CFSR uses a 3Dvar scheme based on the gridpoint statistical interpolation (GSI) data assimilation system while the ocean analyses for SSTs are performed using optimal interpolation. MSLP analyses are available four times per day at 0000, 0600, 1200, and 1800 UTC, and MSLP forecasts are available every hour. Both analyses and forecasts span the period from 1979 to the present.

\section{e. MERRA}

The NASA Modern-Era Retrospective Analysis for Research and Applications (MERRA; Rienecker et al. 2011) uses version 5.2.0 of the Goddard Earth Observing System (GEOS-5) and the GSI data assimilation system (Rienecker et al. 2008) to construct their analysis of observations. The atmospheric system uses a finite volume model, integrated over a grid with horizontal spacings of $2 / 3^{\circ}$ and $1 / 2^{\circ}$ in longitude and latitude respectively (i.e., equivalent to about $55 \mathrm{~km}$ at $45^{\circ}$ of latitude) with 72 sigma vertical levels. The data assimilation used is nearly the same as the one used by the CFSR reanalysis. MSLP analyses are available four times per day at 0000, 0600, 1200 and 1800 UTC; MSLP forecasts are available every hour. Both analyses and forecasts span the period from 1979 to the present.

\section{f. JRA-55}

The Japan Meteorological Agency conducted the second Japanese global atmospheric reanalysis covering the 55 yr from 1958 (JRA-55; Kobayashi et al. 2015). JRA-55 incorporates many improvements compared with the previous version (JRA-25), including a revised longwave radiation scheme, a 4Dvar assimilation scheme, and a variational bias correction scheme for satellite radiances. The JRA-55 system is based on the T319 spectral resolution version (about 55-km grid spacing) of the Japan Meteorological Agency global spectral model (JMA 2013). MSLP analyses are available four times per day at $0000,0600,1200$ and $1800 \mathrm{UTC}$ on a $0.5625^{\circ}$ grid spacing mesh. 


\section{g. $\$ P 2009$}

The SP2009 ECL climatology covers the period between 1970 and 2006 and includes information about the location and dates of lows, a characterization of their associated precipitation, and a categorization of events based on six types of the synoptic-mesoscale environment in which they develop and/or evolve. ECLs are identified over the Tasman Sea in a region delimited by $25^{\circ}-40^{\circ} \mathrm{S}$ and $160^{\circ} \mathrm{E}$ and the Australian coast. Using the information about the date and location of lows, we have calculated the duration of the event by counting the number of consecutive time steps (at 0000 UTC) that a particular low spends in the region of interest.

\section{Methodology}

The methodological approach used here to produce the multiresolution ECL database has three main steps. First, time-varying MSLP fields from the four highresolution reanalyses (CFSR, MERRA, JRA-55, and ERA-I) are aggregated into common lower-resolution grid meshes with grid spacings ranging from 100 to $300 \mathrm{~km}$. Second, a cyclone detection and tracking algorithm is applied to all datasets derived in the first step. Finally, several metrics characterizing the intensity, duration, frequency, and size of lows are calculated for each ECL dataset. A detailed description of each of these steps is given below.

\section{a. Data preprocessing: Spatial regridding}

To analyze the spatial scale dependence of MSLP fields and its impact on ECL identification and tracking, MSLP fields at the reanalyses' native resolution are upscaled into common lower-resolution grid meshes. We first define five regular grid meshes encompassing the same region (whole domain in Fig. 1) but differing in the grid spacing, which ranges between 50 and $300 \mathrm{~km}$. Next, 6-hourly (i.e., 0000, 0600, 1200, and 1800 UTC) instantaneous MSLP fields from each reanalysis at their original resolution (see Table 2 for details) are aggregated in space by calculating the average of the highresolution MSLP values inside lower-resolution grid boxes. Note that this aggregation is only performed when the grid scale of the mesh is not similar to the horizontal spacing of the data in its native grid. That is, when considering the $50-\mathrm{km}$ grid mesh, analyses are performed directly on the native resolution of the data, noting that this varies between reanalyses (see Table 2). Similarly, the $300-\mathrm{km}$ grid mesh does not imply any upscaling for the NCEP1 and NCEP2 reanalyses, which are used at their native resolution $\left(2.5^{\circ} \times 2.5^{\circ}\right)$. Given the larger variations between results for the $50-\mathrm{km}$ and 300-km resolutions, these minor differences in resolution at the extremes are not expected to significantly influence the results.

To illustrate the MSLP smoothing process, Fig. 1 shows an instantaneous MSLP field for an ECL that caused severe coastal impacts in June 2007. Fields are shown for the MERRA reanalysis at its native resolution (Fig. 1a) and a zoom over the cyclone as it appears in the native grid mesh and as obtained by upscaling the native MSLP into the $300-\mathrm{km}$ grid mesh (Fig. 1b; both resolutions are presented on the $50-\mathrm{km}$ grid mesh). Figure 1c shows meridional and zonal cross sections of the cyclone for the native resolution MSLP field and for the various upscaled fields. The cyclone has higher central MSLP and smaller MSLP gradients as the spatial smoothing of the data increases from 50 to $300 \mathrm{~km}$.

A similar experimental setup was used by Blender and Schubert (2000) to study the dependence of cyclones tracks in data with a variety of spatial and temporal resolutions. They use a high-resolution simulation (T106, $2 \mathrm{~h}$ ) as their reference data and their upscaling is performed by linearly interpolating the high-resolution grid instead of averaging in space as we do. The use of the spatial average instead of a linear interpolation to upscale the high-resolution data into coarser grid meshes has the advantage of filtering out the finescale variability, thus better representing the spatial variability simulated by a climate model operating at similar grid spacing.

Finally, the last step of the preprocessing consists of interpolating all datasets, no matter their spatial resolution, into a high-resolution $(50 \mathrm{~km})$ regular grid mesh that encompasses the region of interest. This interpolation is performed using a cubic spline algorithm, which allows for the likely variation on the MSLP fields between grid points, as argued by Murray and Simmonds (1991). Although this high-resolution interpolation does not have much effect when using highresolution reanalyses (see the "Data input and tracking algorithm sensitivity" section in the supplementary material, available online at http://dx.doi.org/10.1175/ JCLI-D-14-00645.s1), it should favor the identification of depressions with scales close to the grid spacing of the low-resolution NCEP1 and NCEP2 reanalyses.

\section{b. Detection and tracking algorithm}

The detection and tracking algorithm is based on the method developed by Browning and Goodwin (2013) and is applied to time-varying fields over the region designated by the black rectangle in Fig. 1a. The main characteristics of the detection algorithm used in this study are described below. 
(a)
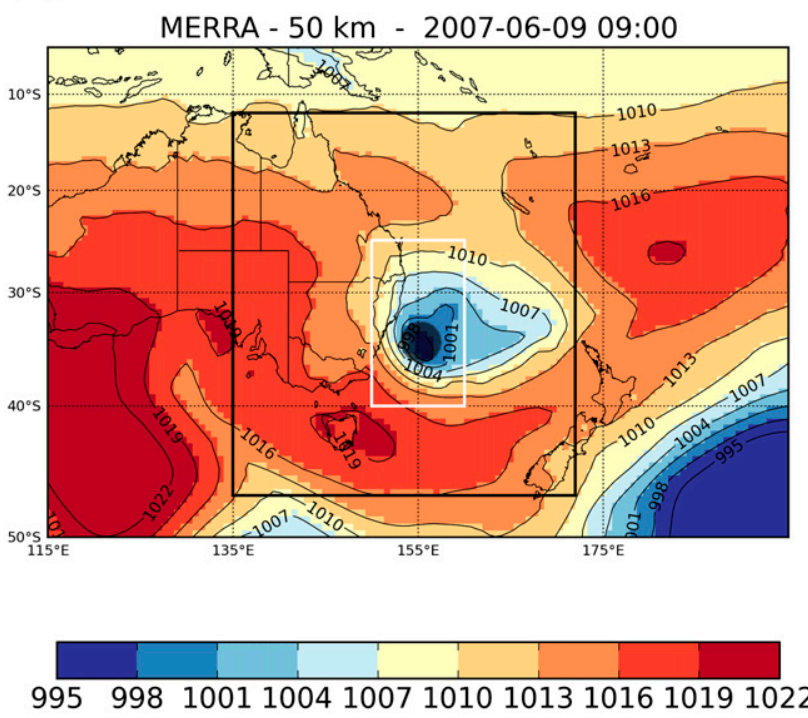

(b)
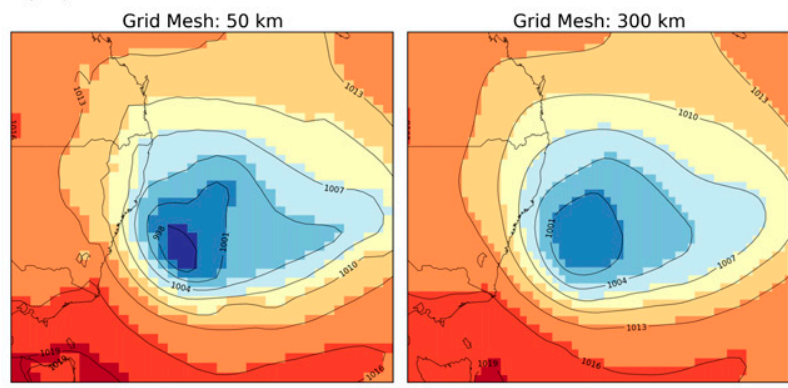

(c)

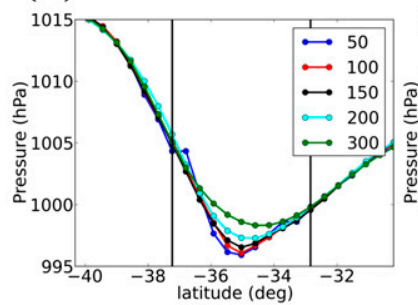

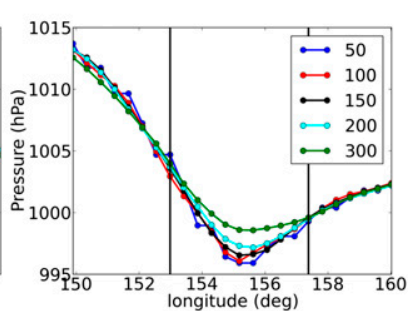

FIG. 1. NASA MERRA instantaneous MSLP field for a severe ECL at 0900 UTC 9 Jun 2007. (a) The region where the tracking is applied (black rectangle) and where the analysis is performed (white rectangle). (b) A zoom of the MSLP field near the cyclone for the native grid mesh and for data upscaled at the 300-km grid mesh. (c) Meridional and zonal cross sections of the MSLP field, through the center of the cyclone, as derived from the data upscaled at different resolutions.

Lows are identified by searching for both a local minima in the MSLP field and a MSLP gradient $(\overline{\nabla p})$ around the local minima that exceeds a given threshold. The use of the local minima criterion implies that only closed lows are identified by the algorithm. The $\overline{\nabla p}$ value is computed by averaging differences between the central MSLP and the MSLP in grid points located within a radius of $200 \mathrm{~km}$ around the central pressure $\left(\overline{\nabla p}_{200}\right)$. While the choice of the $200-\mathrm{km}$ radius is important because it somewhat limits the size of the cyclones detected by the algorithm, the use of other radii (e.g., 100 and $150 \mathrm{~km}$ ) produce essentially the same qualitative results (see Figs. S1 and S2 in the supplementary material). The value of the $200-\mathrm{km}$ MSLP gradient threshold was chosen to be $0.6 \mathrm{hPa}$ per $100 \mathrm{~km}$ and is thus very similar to the threshold used by Browning and Goodwin (2013) of $1 \mathrm{hPa}$ per $1.5^{\circ}$. Using this threshold we obtain a similar number of ECLs compared with SP2009 when considering coarse-resolution data (i.e., between $150-$ and $300-\mathrm{km}$ grid spacing data).

Once lows have been detected (i.e., lows verified by the MSLP minima and MSLP gradient criteria) for individual time steps, cyclone events are generated by grouping lows that are close in both time and space. Tracks are constructed by a nearest neighbor search in the following 6-hourly MSLP field around a cyclone position. The search extends to a maximum distance that depends on the temporal resolution of the data assuming that a cyclone will not move faster than about $60 \mathrm{~km} \mathrm{~h}^{-1}$. The resulting radius of search thus extends to about $360 \mathrm{~km}$ for 6-hourly data. In the case that two different lows are found within a distance of $200 \mathrm{~km}$, only the more intense low is retained.

A number of lows appear to be quasi-stationary features that might be associated either with heat lows or with uncertainties in extrapolating the atmospheric pressure to mean sea level. In this analysis, we filter out some of these quasi-stationary systems by discarding cyclones that move at an average speed smaller than $5 \mathrm{~km} \mathrm{~h}^{-1}$ over the total duration of the event inside the region of interest (i.e., $120 \mathrm{~km}$ for $24-\mathrm{h}$ events). The number of events that are filtered when imposing this condition is generally around $5 \%$ when using highresolution MSLP fields and around 10\% when using low-resolution MSLP fields. Also, for this analysis we only retain events that last at least two consecutive 6-hourly time steps.

As discussed in the introduction, there is a general consensus in the community about the fact that ECLs are mesoscale phenomena (i.e., subsynoptic disturbances; e.g., Holland et al. 1987; Browning and Goodwin 2013) although there is no clear agreement regarding the definition of an ECL. The fact that there is no objective definition of what constitutes an ECL and no perfect dataset that characterizes their climatology makes the 
selection of parameters that define the detection and tracking algorithm ultimately arbitrary. For example, the value of these parameters is sometimes obtained by qualitatively assessing the structure of the identified lows or by trial and error in order to match an observed number of lows. For instance, Pepler and Coutts-Smith (2013) and Browning and Goodwin (2013) used the observation-based SP2009 database as a reference to match the total number of ECLs by choosing the threshold accordingly.

In this study, we have tried to take advantage of the new generation of high-resolution reanalyses and to identify ECL events encompassing a range of intensities, sizes, and durations. Indeed, in order to include most of the low pressure systems that could be regarded as an ECL, we have chosen relatively weak thresholds for the intensity of the lows and the duration of the events. However, we note here that we have performed the analysis using other intensity and duration thresholds and the main conclusions of the article remain unchanged. We include some results in the "Data input and tracking algorithm sensitivity" section in the supplementary material to support the general validity of our results.

\section{c. Evaluation metrics}

From a climatic point of view, the characterization of the average duration, intensity, and size of ECL events together with a measure of their frequency gives a comprehensive description of the climatology of ECLs in a given dataset. The ECL climatology is calculated for the 30-yr period between 1980 and 2009 for which data from all reanalyses are available, although the SP2009 dataset only has data until 2006. Also, ECL statistics are calculated within the region encompassed by $25^{\circ}-40^{\circ} \mathrm{S}$ and $160^{\circ} \mathrm{E}$ and the Australian coast (see white rectangle in Fig. 1), thus only considering maritime ECLs. This relatively small region was selected because it constitutes a common region to reanalyses and the SP2009 dataset.

The intensity of an ECL event is quantified using the mean value of the 200-km MSLP gradient along the track of the cyclone. As shown in the section "Calculating the intensity of events" in the supplementary material, alternative measures of the intensity generally lead to similar results. The duration of the event, when looking at results over a limited area, poses more problems. At least two durations can be calculated for any given event. The first option is to calculate the duration of the event by considering the whole event, no matter whether the low is within the region of interest or not. The second option is to calculate the time that the cyclone spends inside the region of analysis. This second alternative has the advantage of giving longer duration to those events that may have more impact in the region of interest and so we choose this definition in our analysis. A drawback of this last definition is that the "borders" of the region will have on average shorter duration events than, for instance, the center of the region. The mean duration is then calculated by counting the number of time steps the cyclone spends in the region of analysis and multiplying by the time step ( $6 \mathrm{~h}$ for reanalyses and $24 \mathrm{~h}$ for the SP2009 dataset).

Following Rudeva and Gulev (2007), the radius of a given cyclone is estimated by calculating the area inside the so-called last closed isobar. First, we determine the locations for which the first radial derivative of MSLP falls to zero across eight radial lines (north, northeast, east, ...) that pass through the center of the cyclone. The first derivative is calculated using the $50-\mathrm{km}$ grid mesh for a distance of $850 \mathrm{~km}$ from the center. The locations where the derivative is zero are used to determine a "critical" MSLP in each direction and the last closed isobar corresponds to the minimum of all the critical values. In those cases where the derivative is never zero, we set the radius to a maximum value of $850 \mathrm{~km}$ and the critical MSLP corresponds to the MSLP value at $850 \mathrm{~km}$ from the center. Once the MSLP of the last closed isobar is known, distances of this isobar to the center are obtained by interpolation, thus obtaining a radius in each radial direction. We then calculate the area encompassed by the last closed isobar by summing over the triangles obtained using individual radii in different directions. Finally, the radius of the cyclone is obtained by calculating the radius of a circumference that has the same area as the one obtained from the triangle summation.

A more direct comparison between event tracks in different reanalyses can be done using an event-matching algorithm. Following Hodges et al. (2011), we calculate the proportion of events that appear in two given datasets by assuming that an event is the same in any two reanalyses if at least half of the lows composing the event in the reference dataset appear in the other reanalysis (i.e., if half of the lows can be matched in both reanalyses). Two lows are said to be matched when the distance between their centers is smaller than or equal to $300 \mathrm{~km}$ and when they occur within $6 \mathrm{~h}$ of each other. We chose ERA-I as a reference and then we calculated the percentage of ERAI ECL events that are matched in any other reanalysis for every spatial scale:

$$
\begin{aligned}
& \text { matches(dataset, reference) } \\
& =\frac{\mathrm{ECL}(\text { dataset }) \cap \mathrm{ECL}(\text { reference })}{\mathrm{ECL}(\text { reference })} .
\end{aligned}
$$

Calculated as above, the matching rate may be high for those datasets, producing many more events than the 
ERA-I reference. To identify those datasets, we also calculate the critical success index (CSI) score, which takes into account not only the proportion of matches as defined above but also the number of non-matched events:

$$
\operatorname{CSI}(\text { dataset, reference })=\frac{\text { matches }}{\text { matches }+ \text { non-matches }} .
$$

Note that non-matched events can arise either because an event appears in the reference but not in the evaluated dataset or because it appears in the tested dataset but it is absent in the reference. In comparisons using a reference dataset (e.g., temporal-spatial correlations and matching calculations) we arbitrarily chose the ERA-I dataset as the reference. This choice does not imply any value judgement regarding the quality of the data and was made simply based on the fact that ERA-I results appear to be intermediate compared with the other reanalyses.

Statistical confidence of correlation coefficients is tested by assuming that correlations are distributed following a Student's $t$ distribution. At the $95 \%$ confidence level, the significant correlation coefficient can be calculated as $r_{\text {sig }}=\sqrt{1 /\left[1+(N-2) / t_{95, N-2}\right]}$, with $N$ being the size of the sample.

\section{Results}

\section{a. Mean intensity, duration, and size of ECLs}

Figure 2 shows the mean intensity, number, duration, and size of ECLs for the various reanalyses and the SP2009 dataset (whenever possible) as a function of the spatial resolution of the data. Given the subjectivity behind the construction of MSLP maps used to identify ECLs in the SP2009 dataset, it is difficult to associate a specific spatial resolution to the SP2009 dataset. As a consequence, results from the SP2009 dataset are associated simultaneously with all resolutions. All results show annual mean values across all ECL events.

The MSLP gradient averaged over all ECL events (Fig. 2a) shows values between 0.8 and $1.25 \mathrm{hPa}$ $(100 \mathrm{~km})^{-1}$ for the various reanalyses and spatial resolutions. At the $300-\mathrm{km}$ mesh, intensities are very similar $( \pm 5 \%)$ across the four high-resolution reanalyses, with slightly lower intensities when ECLs are identified from reanalyses where the native resolution is coarse. The absolute number of events, mean radius, and mean duration are also very consistent across reanalyses at the $300-\mathrm{km}$ mesh. The SP2009 dataset shows an annual number of events that compares well with the reanalysis results obtained using relatively coarse resolution (i.e., between $200-$ and $300-\mathrm{km}$ spacing) grid meshes. However, it is important to note that the number of events is strongly dependent on the minimum duration threshold, which was chosen to be $12 \mathrm{~h}$ for the reanalyses but corresponds to $24 \mathrm{~h}$ for the SP2009 dataset (see the supplementary material for more discussion).

All high-resolution reanalyses show an increase in the number of events as the horizontal grid mesh increases, with a corresponding increase in the mean intensity as well as a strong decrease in the mean size. However, the resolution dependence varies strongly across datasets, with the average number of ECLs at the native resolution ranging from 22 events for CFSR to about 100 events for MERRA, a factor of 4 . The reanalyses with the highest number of events at native resolutions also have the strongest MSLP gradients, with differences between reanalyses of up to $20 \%$ of the event-mean MSLP gradient at their native resolution. Notably, the mean intensity of ECLs obtained from the CFSR reanalysis shows little sensitivity to changes in the spatial resolution of the MSLP data. In comparison, ERA-I, MERRA, and JRA-55 all show a similar increase in intensity as the spatial resolution increases. At the common $300-\mathrm{km}$ grid mesh, the two low-resolution reanalyses (NCEP1 and NCEP2) show weaker intensities compared to the new high-resolution products.

The mean radius of cyclones (Fig. 2d) shows a large dependence on both the spatial resolution of the data and the choice of the reanalysis. At coarse grid meshes (e.g., $300 \mathrm{~km}$ ), the average cyclone size converges to about $470 \mathrm{~km}$ in the high-resolution reanalyses with the exception of CFSR, which shows a somewhat larger radius (about $525 \mathrm{~km}$ ). Low-resolution reanalyses show larger mean sizes of about $570 \mathrm{~km}$. At higher resolutions there is again large variation between reanalyses, with CFSR showing less sensitivity to changes in resolution than other reanalyses, which suggests CFSR has fewer small cyclones at its native resolution. On the native grid mesh the four reanalyses show large disagreements, with mean sizes varying between $480 \mathrm{~km}$ for CFSR and $\sim 350 \mathrm{~km}$ for MERRA. It is clear from Figs. $2 \mathrm{~b}$ and $2 \mathrm{~d}$ that the number of ECL events is inversely correlated with their average cyclone radius, suggesting that the number of ECL events increases as a reanalysis is able to resolve smaller cyclones. These results also suggest that there are important differences between CFSR and other reanalyses, with substantially fewer small or intense cyclones.

The mean duration of events (Fig. 2c) shows little sensitivity to changes in both the horizontal resolution of the data and the choice of reanalysis, with an annual mean ECL duration of about $22 \mathrm{~h}$. Differences in the 
(a)

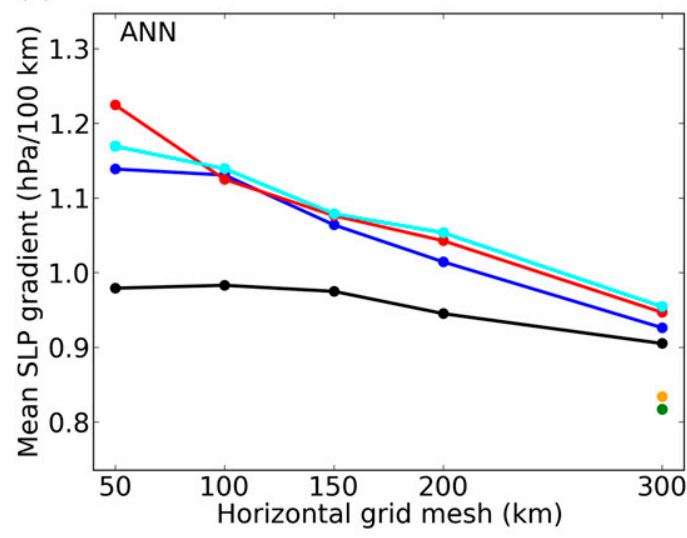

(c)

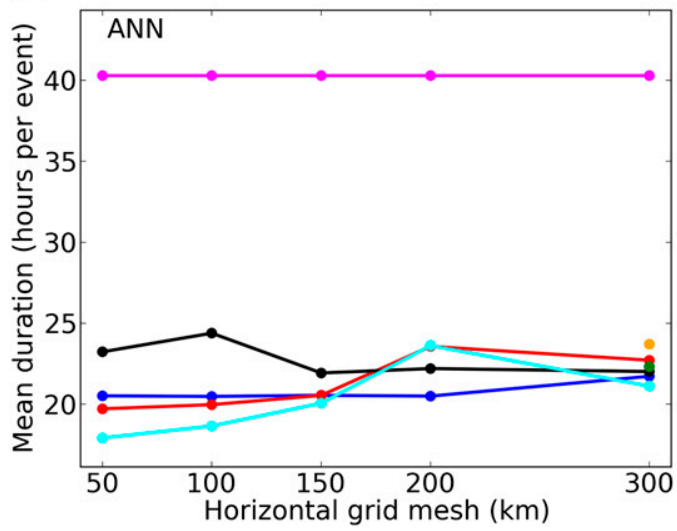

(b)

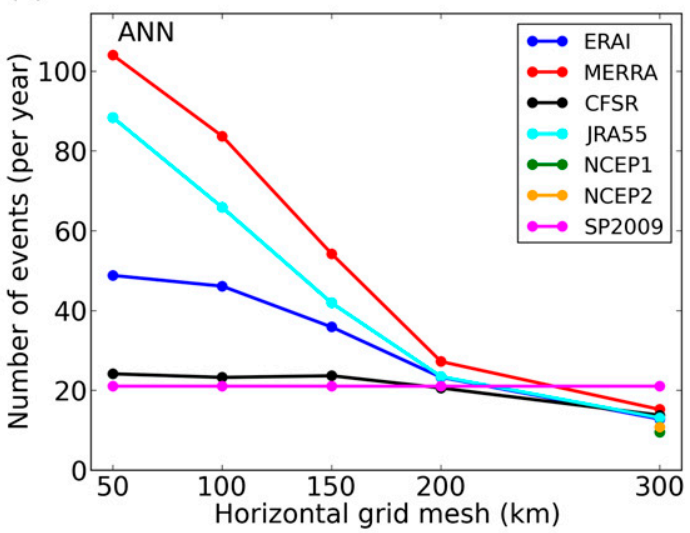

(d)

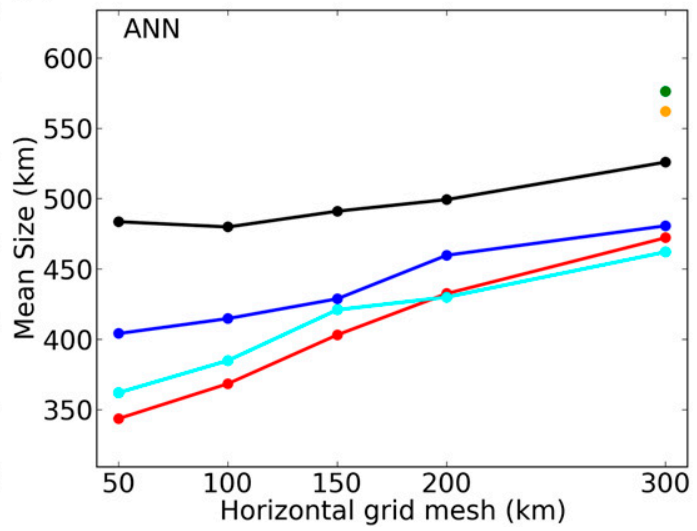

FIG. 2. Annual average of the (a) event-mean 200-km MSLP gradient, (b) number of events, (c) event-mean duration, and (d) event-mean size as a function of the spatial scale. The various datasets are shown in different colors. Low-resolution reanalyses (NCEP1 and NCEP2) and the SP2009 are only available for the 300-km spatial scale.

mean duration across reanalyses are small and generally within $10 \%$. The annual mean duration of events in SP2009 is larger $(40 \mathrm{~h})$. This is largely explained by the fact that SP2009 only samples the MSLP once per day and only identifies ECLs lasting at least $24 \mathrm{~h}$, with the smallest possible duration given as $24 \mathrm{~h}$. As shown in Fig. S3 in the supplementary material, the mean durations obtained in reanalyses when considering ECL events lasting at least $24 \mathrm{~h}$ are very similar to the SP2009 values.

In summary, results in this section show that the ability of the various reanalyses to generate relatively small cyclones is very different with the MERRA product being able to simulate smaller cyclones than any other reanalysis, thus leading to a larger total number of cyclones and a lower mean radius. The smoothing of the reanalysis-derived MSLP fields prior to the identification of ECLs filters out most of the finescale variability and suggests that at coarse scales there is a very strong agreement across the mean characteristics in the various reanalyses, specially across the new high-resolution reanalyses.

\section{b. Distribution of ECL intensity, duration, and size}

Figure 3 a shows the relative frequency of events as a function of the event-averaged $\overline{\nabla p}_{200}$ (i.e., mean intensity) values for the four high-resolution reanalyses at their native resolution in winter. CFSR reanalysis shows a much larger frequency of relatively weak events [i.e., events with $\overline{\nabla p}_{200} \leq 1.0 \mathrm{hPa}(100 \mathrm{~km})^{-1}$ ] and a smaller frequency of more intense events [i.e., events with a $\overline{\nabla p}_{200}>1.0 \mathrm{hPa}(100 \mathrm{~km})^{-1}$ ] than any other high-resolution reanalysis, leading to the lower mean intensity values shown in Fig. 2. The other three reanalyses show a similar distribution of the frequency of events as a function of the mean intensity.

Results for the summer season (Fig. 3d) show that both ERA-I and CFSR present a larger proportion of weak events [i.e., events with $\overline{\nabla p}_{200} \leq 1.0 \mathrm{hPa}(100 \mathrm{~km})^{-1}$ ] and a smaller proportion of more intense events [i.e., 
(a)

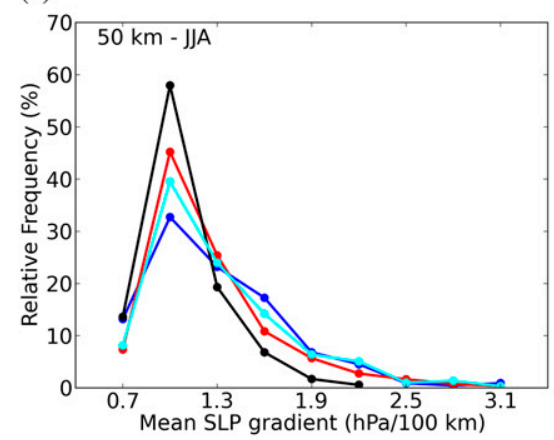

(d)

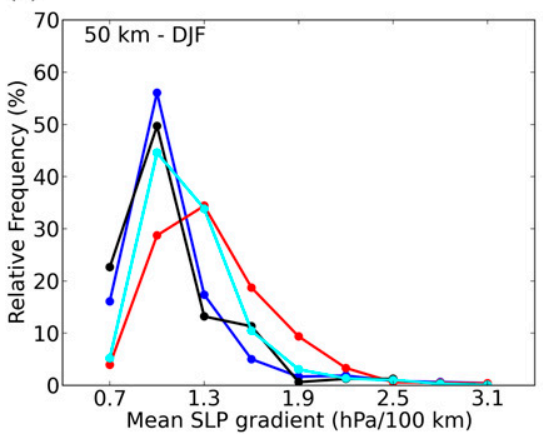

(b)

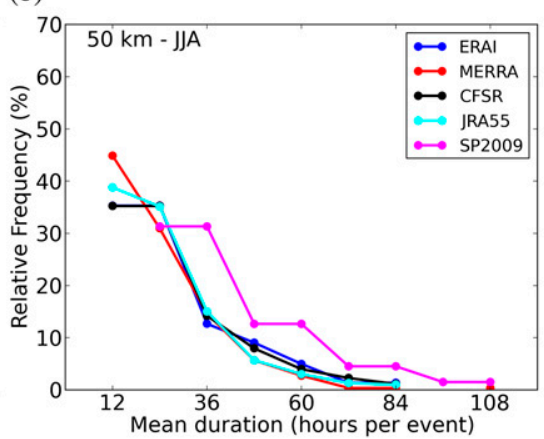

(e)

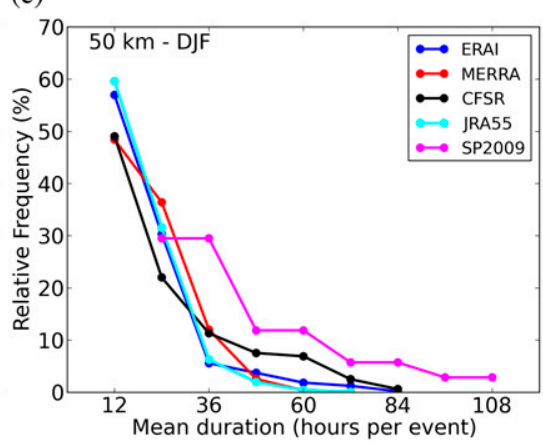

(c)

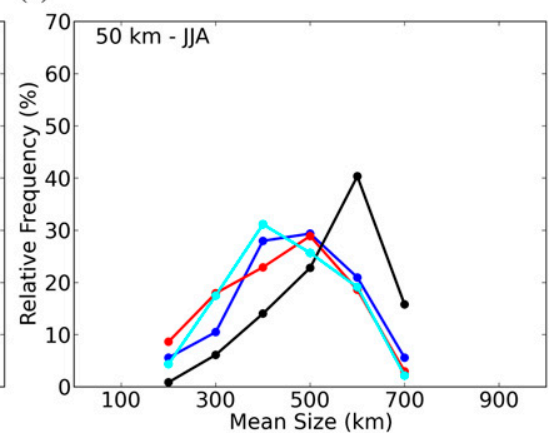

(f)

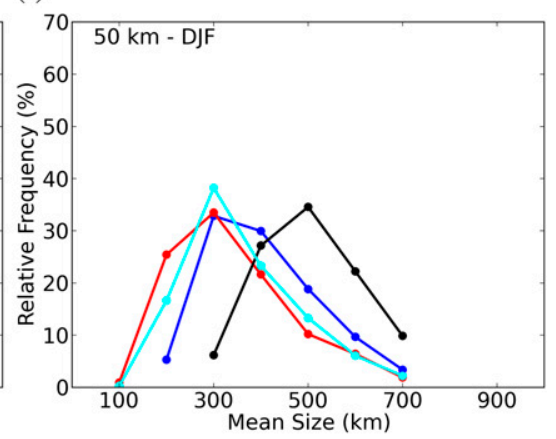

FIG. 3. Frequency distribution of (left to right) the intensity, duration, and size for ECL events identified at the native resolution grid mesh in (top) winter and (bottom) summer. Different colors show the different datasets available in each case. The definition of each metric can be found in section $3 \mathrm{c}$.

events with $\overline{\nabla p}_{200}>1.0 \mathrm{hPa}(100 \mathrm{~km})^{-1}$ ] compared with JRA-55 and particularly compared with the MERRA reanalysis. The much larger proportion of intense cyclones in MERRA and JRA-55 is related to their higher proportion of smaller cyclones, which tend to produce higher values of $\overline{\nabla p}_{200}$ (see the intensity measure discussion in the supplementary material).

The relative frequencies of events as a function of mean duration in winter and summer are presented in Figs. $3 b$ and $3 e$, respectively. In winter, the four highresolution reanalyses produce similar results with the MERRA (CFSR) dataset showing a somewhat larger (smaller) proportion of short-lived events compared to the other reanalyses. Summer results (Fig. 3e) show a very similar distribution of mean duration for ERA-I, JRA-55, and MERRA but CFSR produces a much larger proportion of long-lived ECL events. All highresolution reanalyses show a larger proportion of events that last longer in winter compared to summer, mainly due to a reduction of the proportion of events with mean duration of $12 \mathrm{~h}$ (see Fig. S6b in the supplementary material). As expected from the discussion in the previous section, the SP2009 dataset shows a much larger proportion of long-lived events compared with any other reanalysis.
As was shown for the annual mean values, large differences appear across the four high-resolution reanalyses when considering their mean sizes (Figs. 3c,f). In both seasons but particularly in summer, MERRA and JRA-55 have a larger proportion of small systems compared to CFSR and, to a lesser extent, compared with ERA-I. In summer (Fig. 3e), all reanalyses present a larger proportion of smaller cyclones (see Fig. S6c in the supplementary material), showing that summer ECL events tend to be associated with smaller and shorter-lived cyclones than in winter.

Figure 4 shows the frequency distributions of ECL events relative to mean intensities, durations, and sizes when considering results over the $300-\mathrm{km}$ grid meshes. No matter which characteristic is considered, the four highresolution reanalyses show strong similarities between their distributions. The convergence between reanalyses as the spatial resolution of the MSLP data decreases is thus a relatively robust feature that does not seem to arise from the compensation of errors. The most striking differences between reanalyses appear between the lowresolution reanalyses, NCEP1 and NCEP2, and the highresolution versions, particularly when looking at the mean size of events (Figs. 4c,f) where the low-resolution versions show a larger proportion of larger cyclones. 
(a)

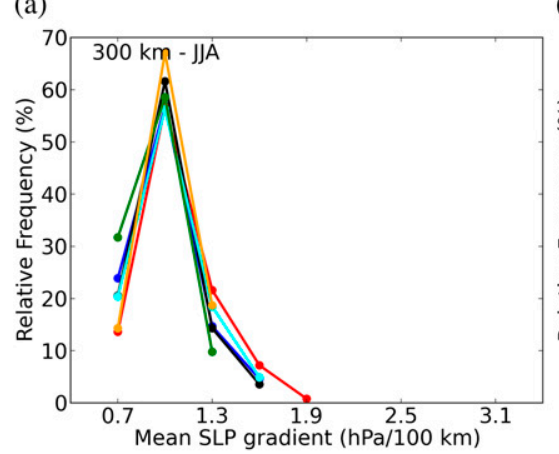

(d)

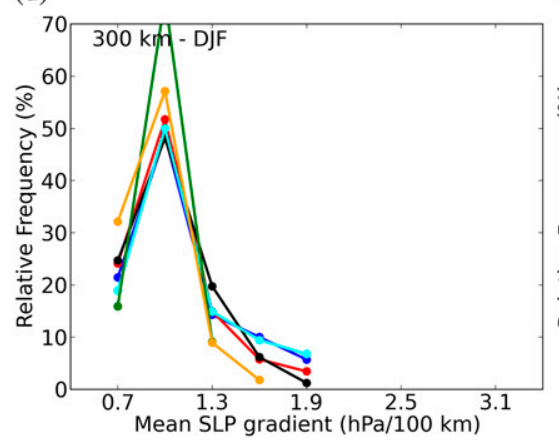

(b)

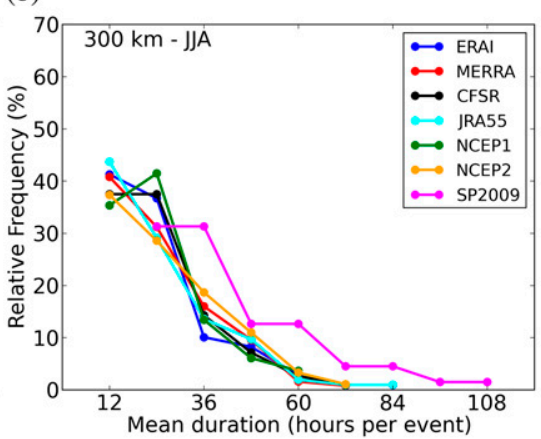

(e)

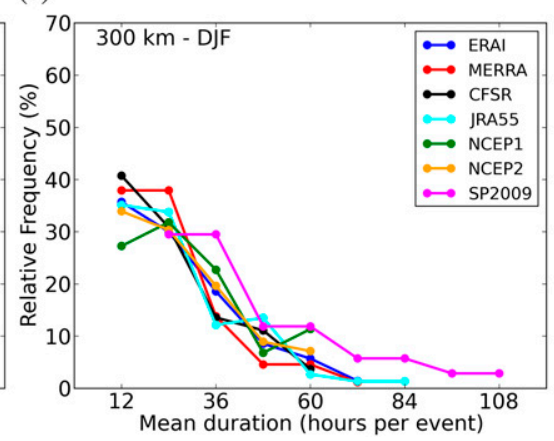

(c)

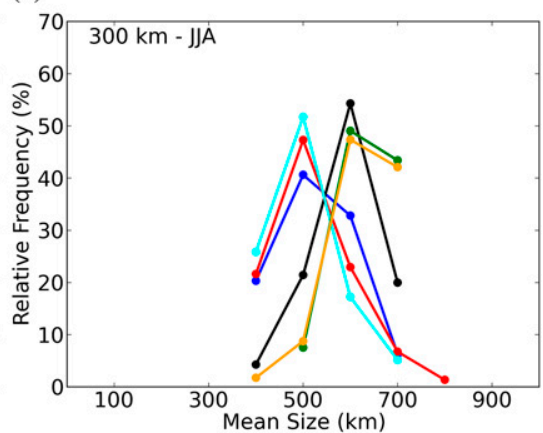

(f)

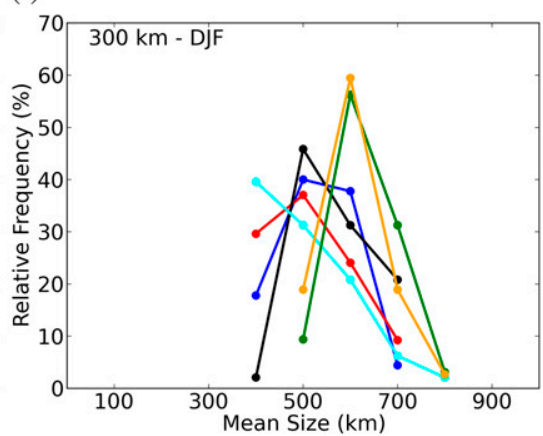

FIG. 4. As in Fig. 3, but for the 300-km spatial scale.

Figures 3a,d and 4a,d show that the intensity of ECLs as measured using the event-mean 200-km MSLP gradient tend be weaker at the $300-\mathrm{km}$ grid mesh than at the native grid mesh, a result that was expected due to the general smoothing of pressure gradients obtained during the averaging process.

Although the relative frequency of events for different mean durations changes little between the native resolution and the $300-\mathrm{km}$ grid mesh results, their mean size distribution does. As expected, the proportion of large cyclones is much larger at the $300-\mathrm{km}$ grid mesh than at the native resolution mesh.

\section{c. Matching individual ECLs}

As described in section $3 \mathrm{c}$, a further evaluation of the similarities of ECLs in the various reanalyses can be done by testing whether or not the same events appear in each of them (i.e., by matching events in a given dataset and a reference, here taken to be ERA-I). Figure 5 shows the percentage of matched events between ERA-I and the other reanalyses as a function of the resolution of MSLP fields used to identify ECLs. In winter (Fig. 5a), results show that no matter the horizontal resolution and the dataset considered, at least $57 \%$ of the ERA-I events also appear in the other datasets. At fine scales, the matching rates are much higher for the ERA-I-MERRA and ERA-I-JRA-55 pairs $(81 \%$ and $77 \%)$ than for the
ERA-I-CFSR pair (63\%). As will be shown later, this is mainly due to the much larger number of events in MERRA and JRA-55 than in CFSR.

In summer (Fig. 5b), the overall matching rate is of $54 \%$, considerably lower than in winter $(74 \%)$. As shown in Figs. 3 and 4, summer events are generally smaller and slightly shorter-lived than winter events regardless of resolution, particularly when considering ERA-I and CFSR datasets. As will be shown later in this section, matching rates are lower for weaker and shorter-lived events, which is a major contributor to the difference in matching rates between summer and winter.

Low-resolution reanalyses show quite high matching rates in both seasons [about $60 \%$ in both June-August (JJA) and December-February (DJF)] but they are systematically lower than matching rates in highresolution reanalyses, suggesting that the upscaling of small-scale features leads to some structural differences on the cyclones at coarser scales.

Figures $5 \mathrm{c}$ and $5 \mathrm{~d}$ show the CSI as a function of reanalysis and spatial resolution in JJA and DJF, respectively. In contrast to the matching results, in winter the CSI values are highest between ERA-I and CFSR and lowest between ERA-I and MERRA. This last result shows that although MERRA and JRA-55 can match more ERA-I events, they do so at the expense of 
(a)

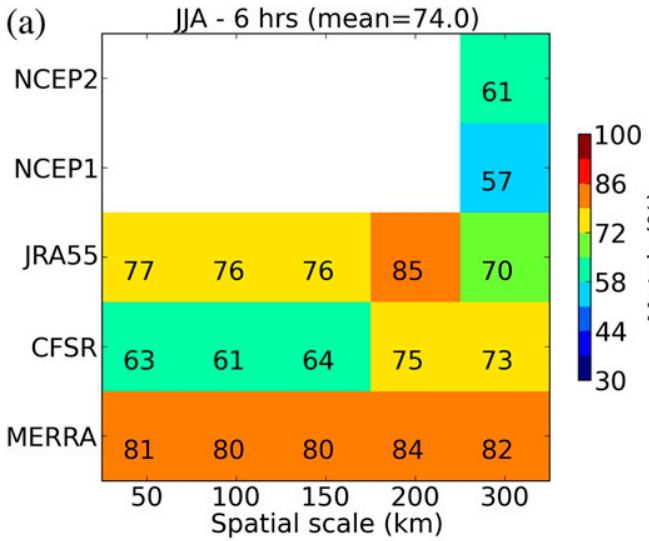

(c)

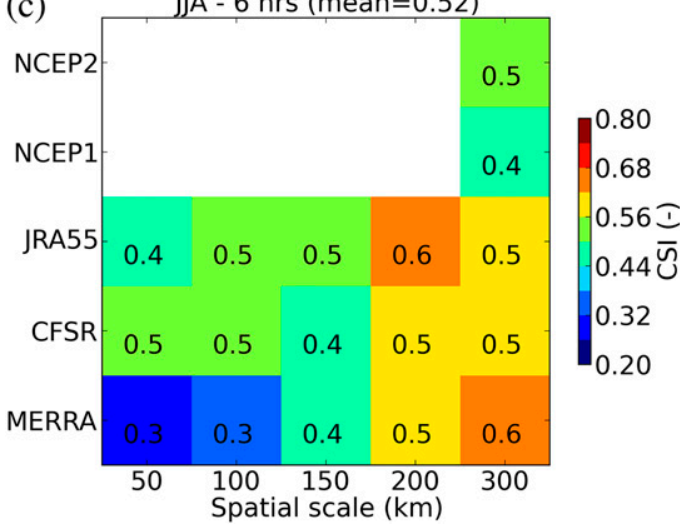

(b)

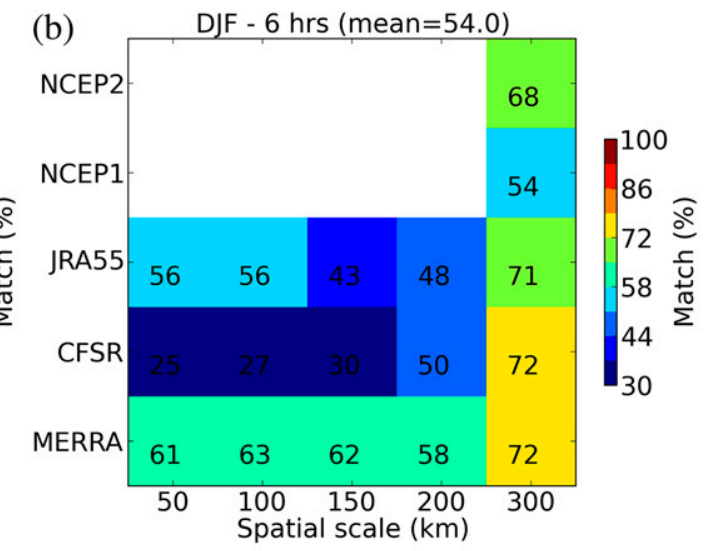

(d)

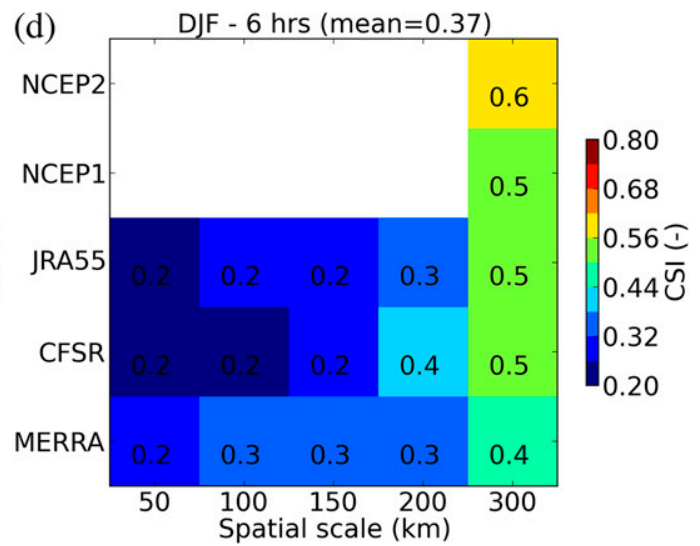

FIG. 5. ECL events (top) matching rates and (bottom) critical success index values (CSI) between ERA-I and the other reanalyses as a function of spatial scales in (left) JJA and (right) DJF. A perfect match between the reference and a given dataset would lead to a $100 \%$ value in matching and a value of 1 in the CSI score.

producing many more events than present in ERA-I. Most high-resolution reanalyses show better agreement with ERA-I as the horizontal resolution of the input MSLP data decreases and this effect is clearer in summer with minimum CSI values of about 0.24 at their native resolution and of about 0.46 at $300-\mathrm{km}$ spatial scale. Low-resolution reanalyses show lower CSI values compared with the high-resolution reanalyses in winter but show similar and even larger CSI values in summer with slightly higher matching rates using NCEP2 than NCEP1.

As found for the matching rates, the overall CSI values are lower in summer (0.37) than in winter $(0.52)$. Also, with the only exception of NCEP1 and NCEP2, all reanalyses show lower CSI values in summer, no matter the spatial resolution of the data.

Figure 6 shows match rates between ERA-I and the other reanalyses when decomposing ERA-I events according to various durations, intensities, and sizes. Results for ECLs detected using the reanalyses at their native resolution (Figs. $6 \mathrm{a}-\mathrm{c}$ ) and using the $300-\mathrm{km}$ grid mesh (Figs. 6d-f) show a general increase of the match rates as the averaged intensity, duration, or size of ECL events increases. Specifically, events lasting more than $36 \mathrm{~h}$ or with an event-mean MSLP gradient larger than $1.3 \mathrm{hPa}(100 \mathrm{~km})^{-1}$ show matching rates always higher than $\sim 70 \%$, no matter the season or the dataset considered. At their native grid meshes, the general increases tend to be larger for CFSR than for MERRA mainly because the MERRA rates are already very high at low intensities, largely due to the much larger number of events as already discussed.

Figure 6 also shows that, for any given intensity, duration, or size, matching rates are always higher in winter than in summer. This is explained by the fact that the chances of having an ECL that is weak, of short duration, and small all at the same time are smaller in winter than in summer. The dependence of matching rates with the size, duration, and intensity of ECLs together with the fact that ECLs are generally smaller and have a shorter duration in summer than in winter as discussed in section $4 \mathrm{~b}$ explain why general 
(a)

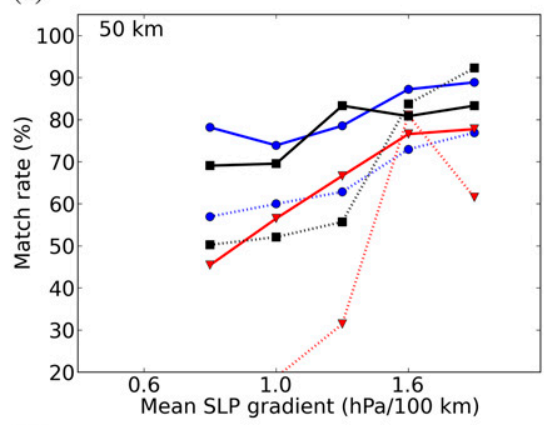

(d)

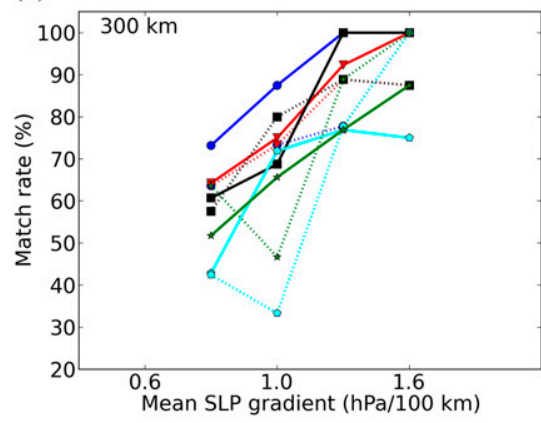

(b)

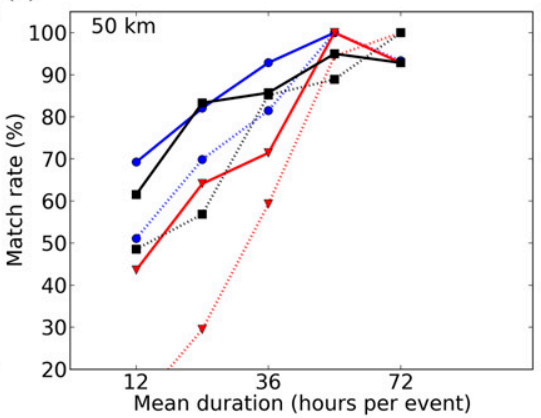

(e)

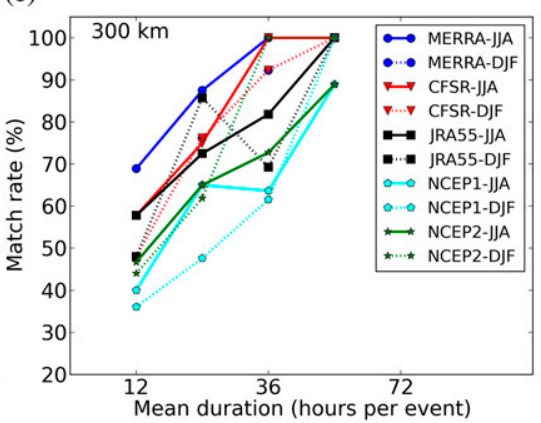

(c)

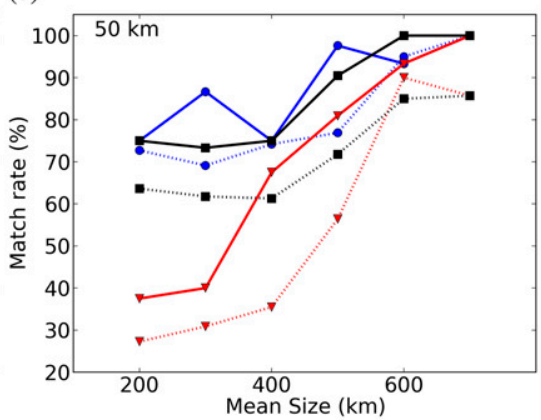

(f)

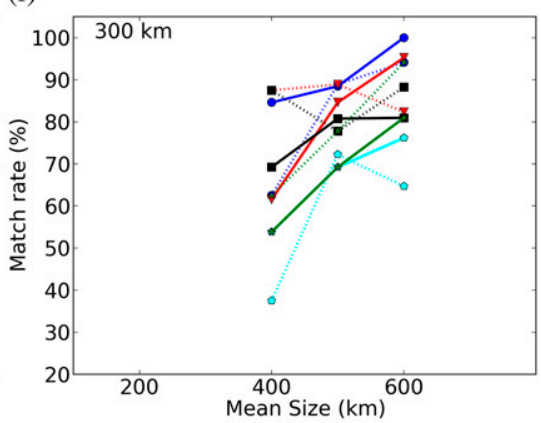

FIG. 6. ECL events matching rates between ERA-I and the other datasets as a function of (left) intensity, (middle) duration, and (right) size of the ERA-I events for (top) the native grid mesh and (bottom) the 300-km grid mesh.

matching rates are much lower in summer than in winter.

\section{d. Monthly and annual variability of ECLs}

Figure $7 \mathrm{a}$ shows the annual cycle of the number of ECL events for all reanalyses and SP2009 at the 300-km grid mesh, normalized by the mean number of events. In all cases there is a maximum frequency in September, with about $50 \%$ more events than the annual average, and a minimum in February with from $30 \%$ to $50 \%$ fewer events that the annual mean number. However, the seasonal cycle using the native grid resolution for the four high-resolution reanalyses (Fig. 7c) is very different, with all but CFSR showing minima in the number of events in winter and a maximum in late spring. As we will show in the next section, this change in seasonality appears to be related with a summer increase in the number of ECLs of the "inland lows" type (see SP2009) in fine-resolution meshes compared with the coarse grid meshes. As shown by SP2009, inland troughs tend to dominate around November explaining the larger number of cyclones in late spring months. The larger number of events in summer in highresolution reanalyses might also be associated with more active convection schemes that can intensify cyclonic systems and their associated MSLP gradients.

To objectively evaluate similarities between ERA-I and the other datasets, Fig. 7e shows the monthly correlations as a function of the ratio of the standard deviations between ERA-I and any other reanalysis for the 50-, 150-, and 300-km spatial scales. No matter the spatial scale and the dataset considered, monthly correlations are generally higher than 0.7 . The exception is CFSR, which shows a very distinct annual cycle at the native resolution and consequently significant correlations compared with ERA-I only at the $300-\mathrm{km}$ grid mesh. Interestingly, the annual cycle of the number of ECL events in the SP2009 dataset is very similar to the one in ERA-I reanalysis only when comparing the $300-\mathrm{km}$ spatial scale (correlation larger than 0.9). This suggests that the kind of events captured by SP2009 is similar to the type of events represented by reanalyses when ECLs are identified using low-resolution MSLP fields.

Figures $7 b$ and $7 d$ show the normalized monthly variability of the mean duration across ECL events for the various reanalyses at the $300-\mathrm{km}$ spatial scale and their native grid mesh. For both spatial scales, the monthly variability of the mean duration is about $20 \%$ of the annual mean value and is thus much smaller than the monthly variability in the number of events. Monthly correlations between reanalyses and ERA-I are generally higher at fine scales due to the stronger annual cycle compared with coarse scale derived ECLs (Fig. 7f). The exception is again the CFSR reanalysis, which shows a distinct seasonality at its native 
(a)
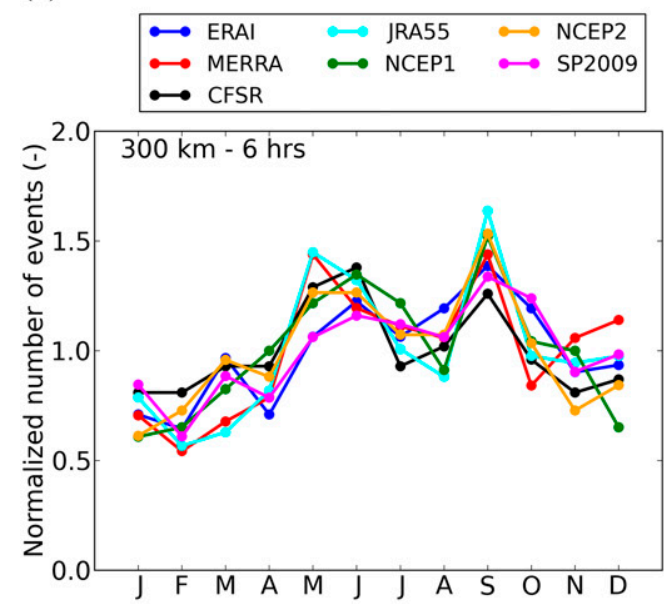

(c)
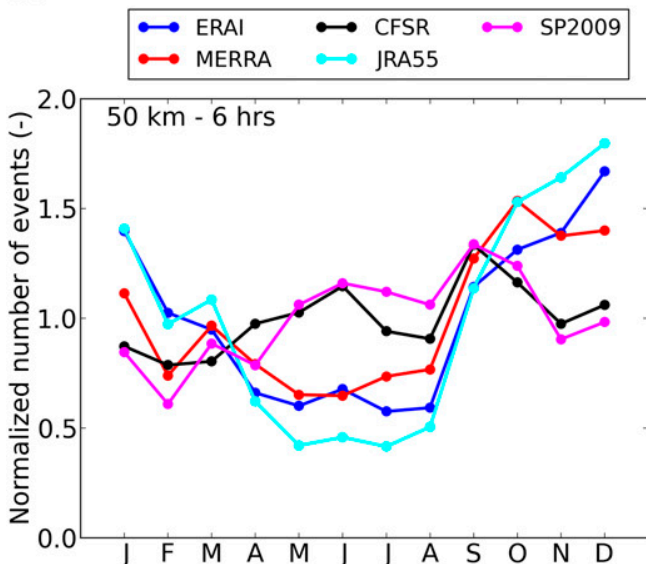

(e)

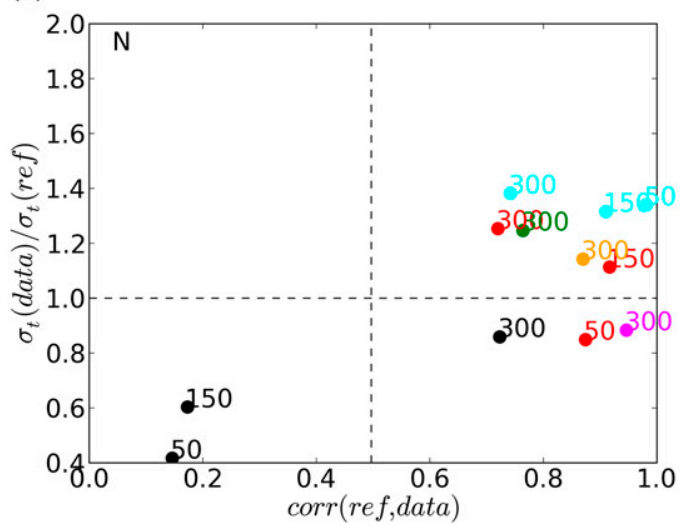

(b)

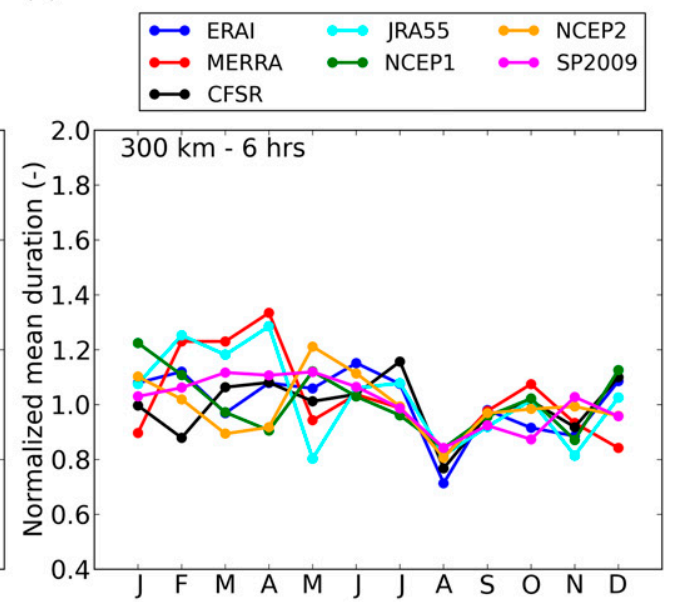

(d)

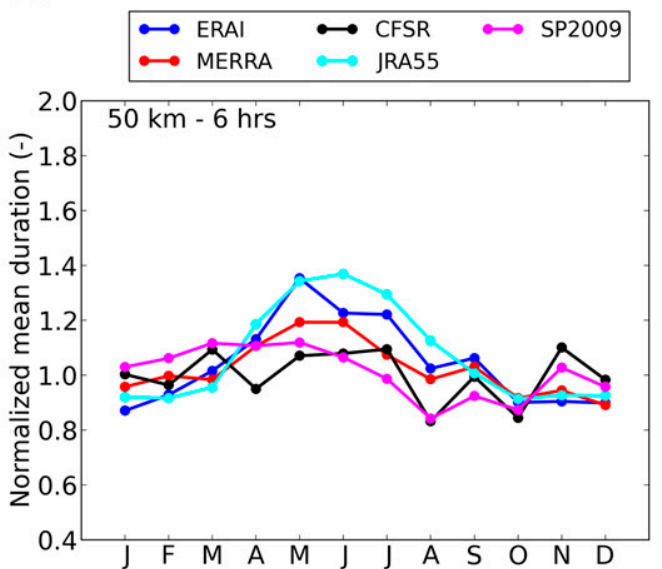

(f)

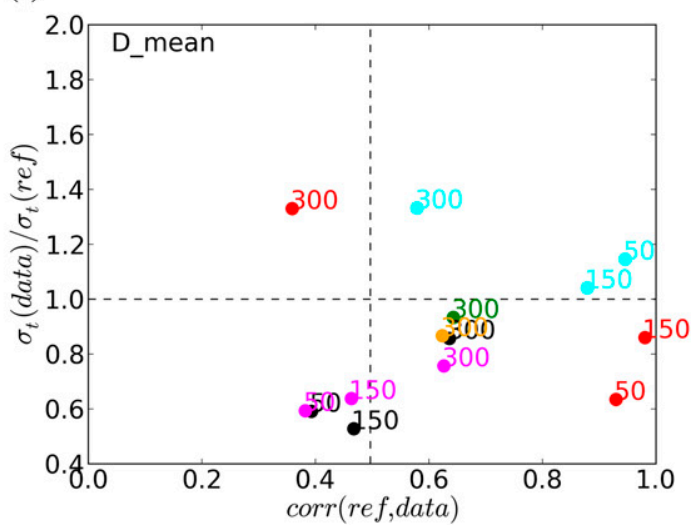

FIG. 7. Normalized annual cycle of the (left) number of events and (right) their mean duration for the various reanalyses at (a),(b) 300-km grid meshes and (c),(d) their native resolution meshes. All values are normalized by the annual mean value. (e),(f) The ratio of standard deviations between ERA-I and any other reanalysis as a function of monthly correlations for the number of events and their mean duration respectively. Different colors denote the different datasets and different numbers denote the different spatial scales. The vertical dashed line shows the value of the significant correlation (see section $3 \mathrm{c}$ for details). 
(a)
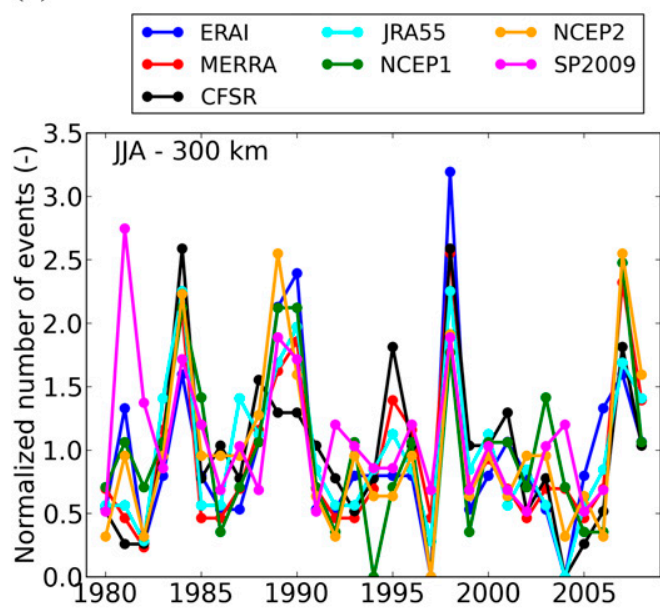

(c)

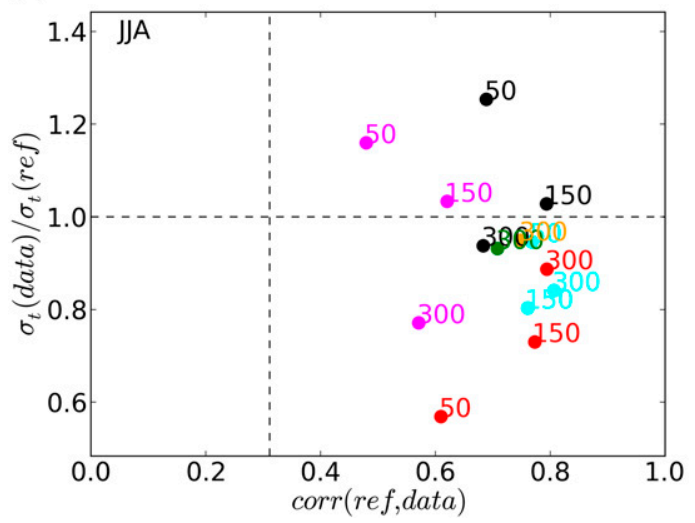

(b)

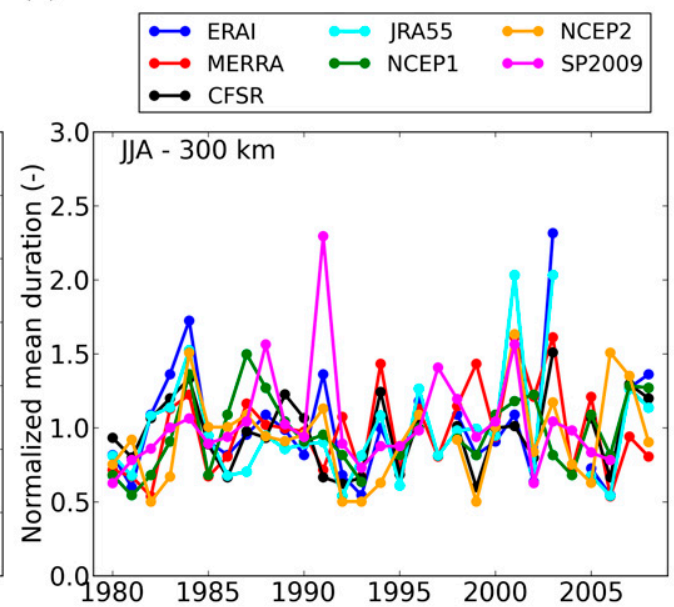

(d)

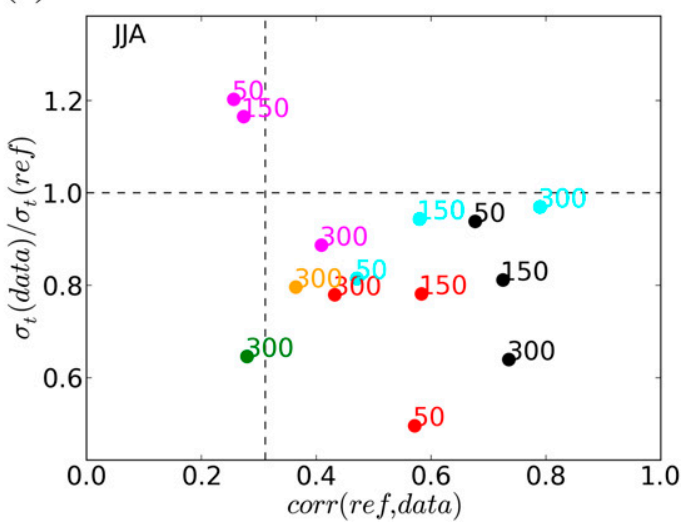

FIG. 8. Normalized interannual variability of (a) the number of events and (b) their mean duration in JJA for the various reanalyses at the $300-\mathrm{km}$ grid mesh. All values are normalized by the winter long-term mean. (c), (d) The ratio of standard deviations between ERA-I and any other reanalysis as a function of annual correlations for the number of events and their mean duration respectively. In (c) and (d), different colors denote the different datasets and different numbers denote the different spatial scales. The vertical dashed line shows the value of the significant correlation (see section $3 \mathrm{c}$ for details).

resolution with monthly correlations smaller than 0.5 . While at the $300-\mathrm{km}$ grid mesh (Fig. 7b) most reanalyses show the longest durations in early autumn and the shortest durations during early spring months, at the native grid mesh (Fig. 7d) high-resolution reanalyses show larger durations in winter and shorter in summer. The annual cycle of mean intensity (not shown) indicates very weak monthly variability with standard deviations varying between 0.03 and 0.05 , suggesting that monthly differences are generally smaller than $10 \%$.

To examine the similarities across datasets in year-toyear variations, Fig. 8 a shows the normalized annual number of events between 1980 and 2009 for the various reanalyses at the $300-\mathrm{km}$ spatial scale in winter. All datasets show a very large interannual variability with some years showing 3 times the long-term mean number of events and other years showing zero events. Reanalyses show similar interannual variations with annual correlations that vary between 0.6 and 0.9 (see Fig. 8c). While the correlations between CFSR and ERA-I are relatively high no matter the spatial scale considered (about 0.7), correlations between MERRA and ERA-I tend to decrease as the grid mesh size increases. Interestingly, the SP2009 dataset shows a similar interannual variability compared with ERA-I reanalysis with a correlation of about 0.6 for the 50-, 150-, and $300-\mathrm{km}$ spatial scale. Given that we use the same SP2009 annual time series to calculate correlations for the three different spatial scales, this result suggests that years with a large (small) number of events are associated with a large (small) number of all kinds of ECLs.

Normalized annual mean durations in winter for the 300-km spatial scale (Fig. 8b) show standard deviations 
(a)
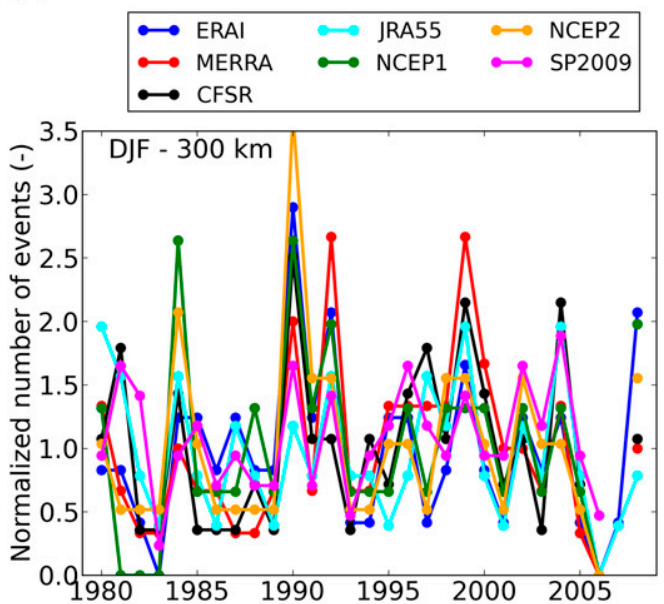

(c)

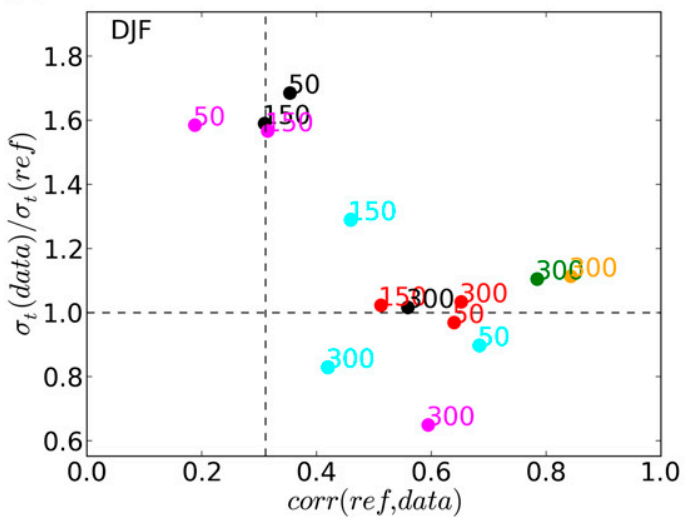

(b)

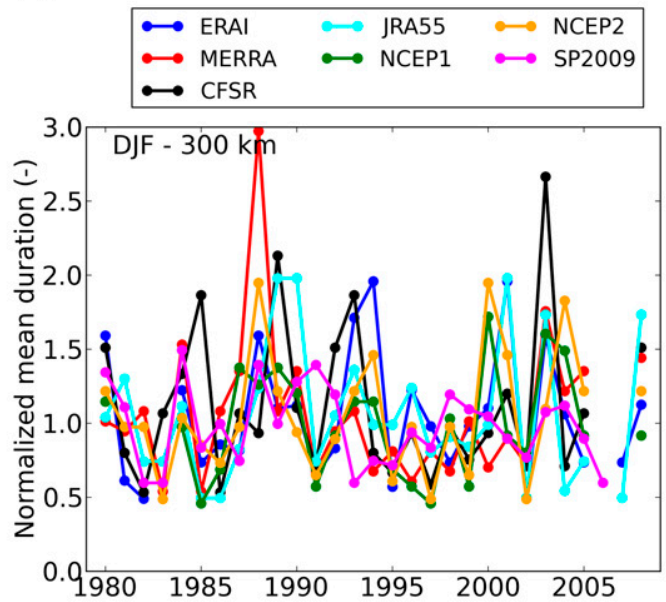

(d)

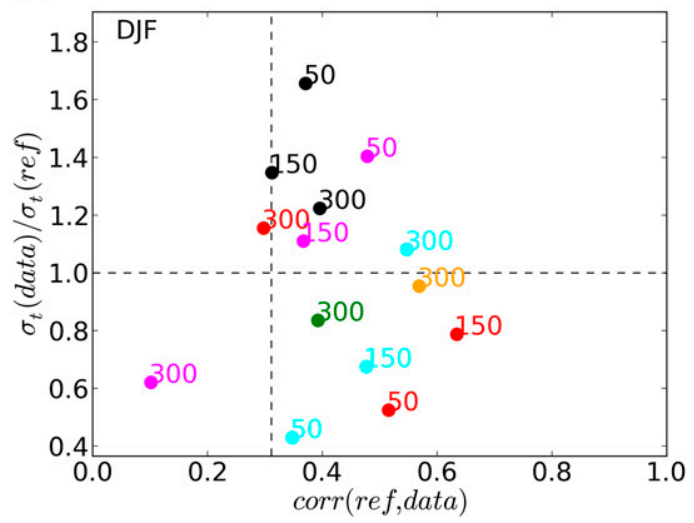

FIG. 9. As in Fig. 8, but for the interannual variability of the number of events and their mean duration in DJF.

varying between 0.23 and 0.28 , thus showing important interannual variability. Correlations between ERA-I and the other reanalyses (Fig. 8d) are generally larger than 0.5 but clearly lower than those obtained for the number of events. For the interannual variability of the mean duration of events, significant correlations between ERA-I and the SP2009 dataset arise only at the 300-km grid mesh.

In summer (Fig. 9), the agreement in the interannual variability between ERA-I and the other reanalyses somewhat deteriorates, as may be expected from the lower matching rates in summer compared to winter. Correlations between the number of events in summer in ERA-I and the other datasets (Fig. 9c) appear to be relatively low with values varying between 0.35 and 0.85 . Again CFSR only shows significant correlations for coarse grid meshes.

\section{e. Spatial variability of ECLs}

To analyze the spatial variability of ECLs over the region of interest, Fig. 10 shows the spatial distribution of the number of events for the ERA-I dataset in winter (left panels) and summer (right panels) for results obtained at their native resolution (top panels) and with the $300-\mathrm{km}$ (middle panels) grid meshes. Regardless of the resolution of the data, in winter ERA-I shows a clear north-south gradient on the number of ECLs with between 2 and 10 times more cyclones in the south compared to the northern part of the region of analysis. This north-south gradient is stronger at the $300-\mathrm{km}$ grid mesh due to the greater proportion of large extratropical cyclones at this resolution compared to the reanalysis native grid mesh.

To examine differences across reanalyses between the spatial distributions of ECL events over the region of analysis, the spatial correlations between ERA-I and the other reanalyses are shown in Fig. 10e for winter results. Correlations are generally higher than 0.7 , indicating a strong agreement between reanalyses regardless of spatial resolution of the MSLP fields used to identify ECLs. Moreover, the magnitude of the spatial variability appears to be quite similar in all datasets with ratios of 
(a)

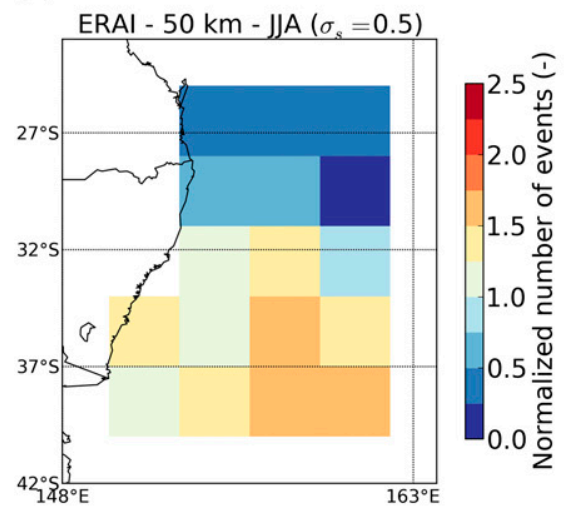

(c)

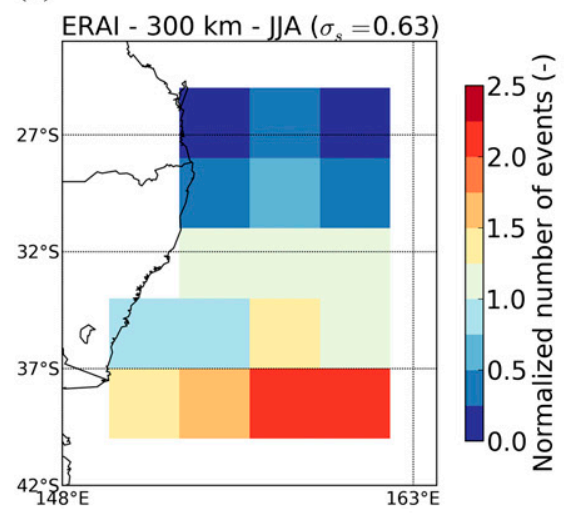

(e)

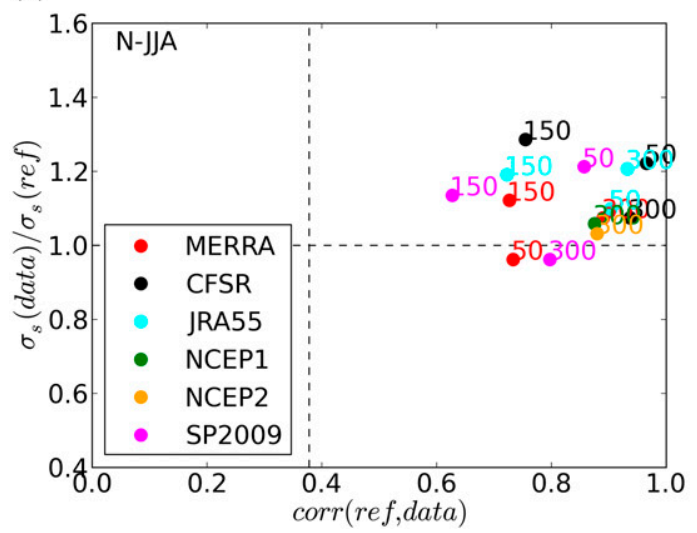

(b)

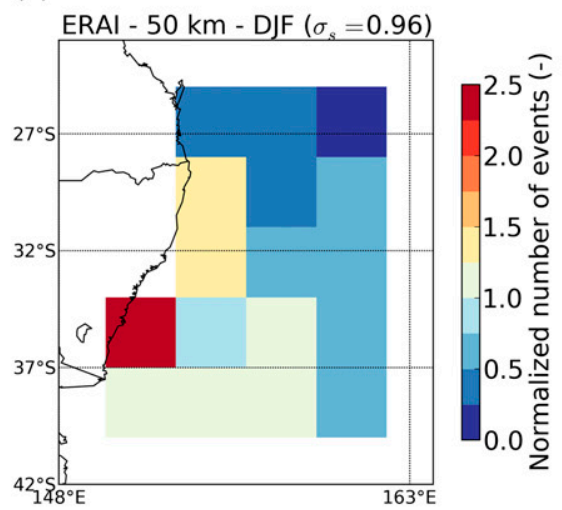

(d)

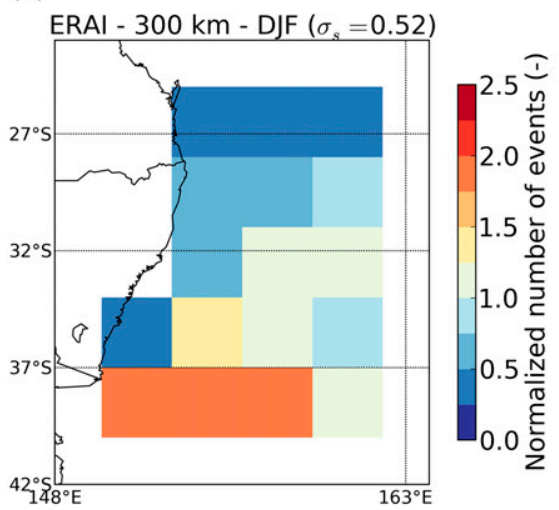

(f)

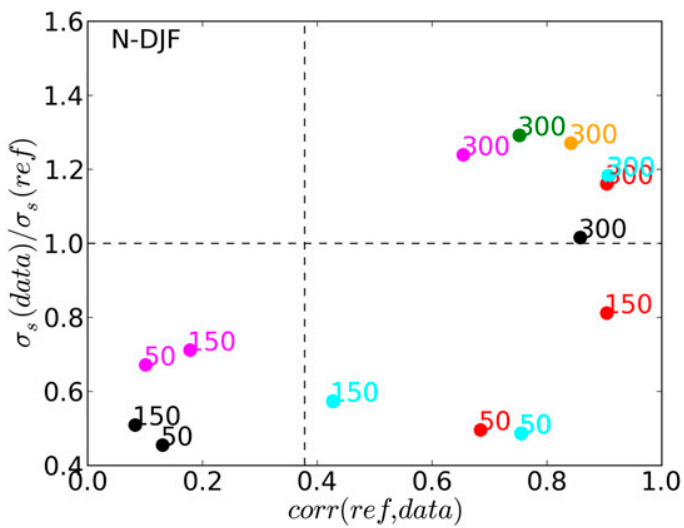

FIG. 10. Normalized number of lows for the (top) native and (middle) 300-km grid mesh results in (left) JJA and (right) DJF. All values are normalized by the seasonal mean number of events over the whole region. (bottom) The ratio of the standard deviations between ERA-I and any other reanalysis as a function of spatial correlations for the different resolution datasets. The vertical dashed line shows the value of the significant correlation (see section $3 \mathrm{c}$ for details).

standard deviations generally between 0.9 and 1.3. As found for the interannual variability in winter, the SP2009 dataset shows a similar spatial distribution of ECL events compared with ERA-I, no matter the spatial resolution of the MSLP fields (i.e., correlation of about 0.8).

In summer, the north-south gradient is also evident (but weaker) at the 300-km spatial scale (Figs. 10d), but less clear at the native resolution (Fig. 10b). Instead, at fine scales there is an increase in the number of cyclones near the eastern coast of Australia, which appears to be consistent with a larger number of ECL events of the "inland trough" type that develop within coastal surface troughs (see the annual cycle and the spatial distribution of inland trough lows in SP2009). Spatial correlations 
between ERA-I and the other datasets (Fig. 10f) slightly deteriorate compared with winter results but are still high with values generally higher than 0.7 , suggesting that most datasets share a similar spatial distribution of ECLs. As found for the comparisons of the interannual variability in summer, CFSR and the SP2009 dataset show significant spatial correlations with ERA-I only at coarse spatial scales.

\section{Discussion and conclusions}

We have compared the climatology of maritime cyclones to the east of the Australian east coast using four high-resolution (NASA MERRA, NCEP CFSR, JRA55, and ERA-I) and two low-resolution (NCEP-NCAR and NCEP-DOE) reanalyses and, whenever possible, the ECL observation-based dataset from SP2009. The methodological approach used to compare the various datasets was designed to isolate differences related to the distinct horizontal resolutions from those related to the assimilation systems structure and general quality (i.e., assimilation scheme, type of model, number of observations assimilated, etc.). For that purpose, 6-hourly MSLP fields from the four high-resolution reanalyses were first regridded into several common lower-resolution grid meshes prior to the application of a single algorithm to identify and track lows. The tracking algorithm was specially tuned to identify relatively small cyclones and thus allows the exploration of subsynoptic-scale cyclones on the new generation of reanalyses.

We have shown that several characteristics of ECLs as derived from reanalyses are in agreement with the SP2009 database. In particular, monthly, interannual, and spatial variability of the number of events and their duration appear to be similar in SP2009 and the reanalysis-derived climatologies when using lowresolution MSLP fields to identify ECLs. Results suggest that ECL events in the SP2009 database are more in agreement with ECLs obtained using coarse-resolution MSLP fields from reanalyses. Although interannual and spatial variability show little dependence with resolution in winter, summer results show significant differences depending on the resolution of the MSLP data, thus indicating important differences between the ECL climatology in SP2009 and in reanalyses at their native resolution.

The assessment of ECL results obtained using several reanalysis products after regridded at different horizontal resolutions leads to the following main results:

- When identifying ECLs using MSLP fields at coarse resolutions $(\Delta x \approx 300 \mathrm{~km})$, the modern reanalyses produce very similar climatologies of ECLs with differences of mean intensities, frequencies, durations, and sizes within $5 \%$ of the mean values. Furthermore, we showed that the agreement in the mean values of the various quantities arises from a very similar representation of their frequency distributions. The analysis of the matching of individual events shows that more than $70 \%(71 \%)$ of winter (summer) cyclones in ERA-I also appear in CFSR and MERRA and these rates largely increase when discarding relatively weak, small, and/or short-duration events. Moreover, we found that reanalyses show a high degree of consistency in their representation of the seasonal, annual, and spatial variability of ECLs. These results are in agreement with results from Dowdy et al. (2013), who found very similar climatologies in three different reanalyses when using a largescale diagnostic to evaluate the risk of ECL formation.

- Results obtained using the low-resolution NCEP1 and NCEP2 reanalyses generally agree well with those obtained using the newer, higher-resolution products (i.e., ERA-I, MERRA, JRA-55, or CFSR) when compared at the same resolution. However, NCEP1 and NCEP2 systematically show lower mean intensities and larger mean size of cyclones, suggesting that there are some consistent improvements in the largescale MSLP fields that arise from the upscaling of small-scale features.

- Although all reanalyses show similar results at coarse scales, they show very distinct sensitivities to changes in the horizontal resolution of the data. MERRAJRA-55, ERA-I, and CFSR show a very strong, moderate, and weak sensitivity respectively. All reanalyses show an increase in the number of cyclones as the horizontal grid spacing of the MSLP data decreases from 300 to $50 \mathrm{~km}$ because a higher-resolution grid mesh allows to resolve smaller and consequently more cyclones. This truncation effect is more pronounced in summer than in winter because of the larger proportion of small cyclones in the former season.

- An increase on the number of cyclones with increasing horizontal resolution was also observed by Blender and Schubert (2000) and Tilinina et al. (2013). As in Blender and Schubert (2000) the sensitivity to horizontal resolution considered in this study only reflects the effect of truncation on the MSLP fields.

- When identifying ECLs using MSLP fields at high resolutions $(\Delta x \approx 50 \mathrm{~km})$, differences in the frequency, intensity, and size between the high-resolution reanalyses are very large with the largest discrepancies arising between MERRA and CFSR. In both winter and summer but especially in the later, MERRA 
shows a larger number of short-lived, strong, and small cyclones than any other reanalyses. Specifically, MERRA shows more than 4 times the number of events and less than half the mean radius of cyclones compared with the CFSR reanalysis. Based on cyclones tracked over the whole NH, Tilinina et al. (2013) also found that MERRA produces a larger number of cyclones compared to any other reanalyses together with a larger proportion of very strong, short, and rapidly intensifying cyclones. The agreement between our results and those from Tilinina et al. (2013) suggests that these differences are related to some intrinsic difference in the models use to generate the reanalyses and are not related to the specific region of analysis.

- Results obtained from a number of sensitivity tests (see the supplementary material) performed using different MSLP gradient and duration thresholds, using different interpolation methods, and using forecast fields instead of analyses suggest that differences in the number of events across the reanalyses do not appear to be related to the assimilation process or the exact formulation of the algorithm used to identify and track cyclones. Neither of these differences appears to be related with the original resolution of the models because the reanalysis produced using the highest-resolution model (CFSR; see Table 1) appears to be the least sensitive to changes in the resolution of the data.

Rather, a few alternative hypotheses can be considered to explain the differences across the high-resolution reanalyses. The much larger sensitivity observed in the MERRA reanalyses could be associated with the finite volume method used to solve the equations of state compared with the spectral method used in the other high-resolution reanalyses. This hypothesis was suggested by Tilinina et al. (2013) as the cause of the larger number of cyclones and their larger intensity in MERRA relative to other products. Also, differences in cumulus schemes used by the various reanalysis models could lead to important differences in ECLs statistics, particularly in the summer season when cumulus schemes are more active. Finally, the distinct behavior of the CFSR reanalysis product could be associated with the use of a coupled atmosphere-ocean model instead of a standalone atmospheric model as in the other reanalyses. Clearly, further work is needed to determine the sources of the differences across reanalyses.

- ECL climatologies derived from reanalyses show significant monthly, annual, and spatial variability, particularly when looking at the frequency of events and, to a lesser extent, their mean duration. ECL statistics derived from the various reanalyses generally agree quite well in the representation of the magnitude of the variability and they show similar temporal and spatial patterns of variability. ECL statistics derived from CFSR at its native resolution show however some significant differences in the annual cycle and in the interannual and spatial variability compared with the other high-resolution products, particularly in summer season.

In agreement with the study by Dowdy et al. (2013), this study shows that for relatively large-scale cyclones, different reanalyses give a very consistent picture of the ECLs properties and their variability over the eastern coast of Australia. As a consequence, the use of a single reanalysis to study characteristics of maritime cyclones over the eastern coast of Australia may be well justified when looking at relatively large cyclones (e.g., those obtained after spatially smoothing MSLP fields) particularly when considering one of the new high-resolution reanalyses (i.e., MERRA, JRA-55, CFSR, or ERA-I).

However, when looking at subsynoptic-scale cyclones in reanalyses at their original resolution, uncertainties about their frequency, intensity, duration, and size are very large and it is not clear which reanalysis, if any, gives a better description of ECL events.

The study also highlights the advantages of using reanalysis products together with an automatic algorithm to detect and track cyclones because it allows the study of a large variety of physical properties of ECL events and to extend results to other regions and periods. Clearly, further work is needed in order to evaluate the finescale information in reanalyses and to better understand to what extent this information can be used to assess small-scale cyclones in other products.

Acknowledgments. This work is supported by funding from the NSW Environmental Trust and the NSW Office of Environment and Heritage for the ESCCIECL project through the Australian Research Council Grant LP120200777. Jason Evans was supported by the Australian Research Council Future Fellowship FT110100576. Research was also supported by the Australian Research Council Centre of Excellence for Climate System Science, Grant CE110001028. The authors would like to acknowledge Stuart Browning for his provision of the identification and tracking scheme code and of advice on running it.

\section{REFERENCES}

Blender, R., and M. Schubert, 2000: Cyclone tracking in different spatial and temporal resolutions. Mon. Wea. Rev., 128, 377-384, doi:10.1175/1520-0493(2000)128<0377:CTIDSA > 2.0.CO;2. 
Browning, S. A., and I. D. Goodwin, 2013: Large-scale influences on the evolution of winter subtropical maritime cyclones affecting Australia's east coast. Mon. Wea. Rev., 141, 2416-2431, doi:10.1175/MWR-D-12-00312.1.

Dee, D. P., and Coauthors, 2011: The ERA-Interim reanalysis: Configuration and performance of the data assimilation system. Quart. J. Roy. Meteor. Soc., 137, 553-597, doi:10.1002/qj.828.

Dowdy, A. J., G. A. Mills, B. Timbal, and Y. Wang, 2013: Changes in the risk of extratropical cyclones in eastern Australia. J. Climate, 26, 1403-1417, doi:10.1175/JCLI-D-12-00192.1.

Evans, J. P., F. Ji, C. Lee, P. Smith, D. Argüeso, and L. Fita, 2014: Design of a regional climate modelling projection ensemble experiment-NARCliM. Geosci. Model Dev., 7, 621-629, doi:10.5194/gmd-7-621-2014.

Griffies, S. M., M. J. Harrison, R. C. Pacanowski, and A. Rosati, 2004: A technical guide to MOM4. GFDL Ocean Group Tech. Rep. 5, NOAA/GFDL, 342 pp.

Hodges, K. I., R. W. Lee, and L. Bengtsson, 2011: A comparison of extratropical cyclones in recent reanalyses ERA-Interim, NASA MERRA, NCEP CFSR, and JRA-25. J. Climate, 24, 4888-4906, doi:10.1175/2011JCLI4097.1.

Holland, G. J., A. H. Lynch, and W. Leslie, 1987: Australian east-coast cyclones. Part I: Synoptic overview and case study. Mon. Wea. Rev., 115, 3024-3036, doi:10.1175/1520-0493(1987)115<3024: AECCPI $>2.0 . \mathrm{CO} ; 2$.

Hopkins, L. C., and G. J. Holland, 1997: Australian heavy-rain days and associated east coast cyclones: 1958-92. J. Climate, 10, 621-635, doi:10.1175/1520-0442(1997)010<0621:AHRDAA > 2.0.CO;2.

JMA, 2013: Numerical weather prediction (NWP) by the Japan Meteorological Agency (JMA). [Available online at http://www.jma go.jp/jma/jma-eng/jma-center/nwp/nwp-top.htm.]

Kalnay, E., and Coauthors, 1996: The NCEP/NCAR 40-Year Reanalysis Project. Bull. Amer. Meteor. Soc., 77, 437-471, doi:10.1175/1520-0477(1996)077,0437:TNYRP.2.0.CO;2.

Kanamitsu, M., W. Ebisuzaki, J. Woollen, S.-K. Yang, J. J. Hnilo, M. Fiorino, and G. L. Potter, 2002: NCEP-DOE AMIP-II Reanalysis (R-2). Bull. Amer. Meteor. Soc., 83, 1631-1643, doi:10.1175/BAMS-83-11-1631.

Kobayashi, S., and Coauthors, 2015: The JRA-55 reanalysis: General specifications and basic characteristics. J. Meteor. Soc. Japan, 93, 5-48, doi:10.2151/jmsj.2015-001.
Mills, G. A., R. Webb, N. E. Davidson, J. Kepert, A. Seed, and D. Abbs, 2010: The Pasha Bulker East Coast low of 8 June 2007. Centre for Australia Weather and Climate Research Tech. Rep. 23, 62 pp.

Murray, R. J., and I. Simmonds, 1991: A numerical scheme for tracking cyclone centres from digital data. Part I: Development and operation of the scheme. Aust. Meteor. Mag., 39, $155-166$.

Pepler, A. S., and A. Coutts-Smith, 2013: A new, objective, database of East Coast lows. Aust. Meteor. Oceanogr. J., 63 (4), 461-472.

—, A. Di Luca, F. Ji, L. V. Alexander, J. P. Evans, and S. C. Sherwood, 2015: Impact of identification method on the inferred characteristics and variability of Australian East Coast lows. Mon. Wea. Rev., 143, 864-877, doi:10.1175/ MWR-D-14-00188.1.

Rienecker, M. M., and Coauthors, 2008: The GEOS-5 data assimilation system documentation of versions 5.0.1 and 5.1.0, and 5.2.0. NASA Tech. Rep. NASA/TM-2008-104606, 92 pp.

— , and Coauthors, 2011: MERRA: NASA's Modern-Era Retrospective Analysis for Research and Applications. J. Climate, 24, 3624-3648, doi:10.1175/JCLI-D-11-00015.1.

Rudeva, I., and S. K. Gulev, 2007: Climatology of cyclone size characteristics and their changes during the cyclone life cycle. Mon. Wea. Rev., 135, 2568-2587, doi:10.1175/MWR3420.1.

Saha, S., and Coauthors, 2010: The NCEP Climate Forecast System Reanalysis. Bull. Amer. Meteor. Soc., 91, 1015-1057, doi:10.1175/ 2010BAMS3001.1.

Simmonds, I., and K. Keay, 2000: Mean Southern Hemisphere extratropical cyclone behavior in the 40-year NCEPNCAR reanalysis. J. Climate, 13, 873-885, doi:10.1175/ 1520-0442(2000)013<0873:MSHECB > 2.0.CO;2.

Speer, M. S., P. Wiles, and A. Pepler, 2009: Low pressure systems off the New South Wales coast and associated hazardous weather: Establishment of a database. Aust. Meteor. Oceanogr. J., 58, 29-39. [Available online at http://web.maths.unsw. edu.au/ mss/index_files/speeretal_published.pdf.]

Tilinina, N., S. K. Gulev, I. Rudeva, and P. Koltermann, 2013: Comparing cyclone life cycle characteristics and their interannual variability in different reanalyses. J. Climate, 26, 6419-6438, doi:10.1175/JCLI-D-12-00777.1. 